\title{
On the Cauchy problem for the magnetic Zakharov system
}

\author{
Boling Guo ${ }^{1}$ Jingjun Zhang ${ }^{2,3 *}$ Chunxiao Guo ${ }^{2}$ \\ ${ }^{1}$ Institute of Applied Physics and Computational Mathematics \\ P. O. Box 8009, Beijing, China, 100088 \\ ${ }^{2}$ The Graduate School of China Academy of Engineering Physics \\ P. O. Box 2101, Beijing, China, 100088 \\ ${ }^{3}$ College of Mathematics and Information Engineering \\ Jiaxing University, Zhejiang, China, 314001
}

\begin{abstract}
In this paper, we study the Cauchy problem of the magnetic type Zakharov system which describes the pondermotive force and magnetic field generation effects resulting from the non-linear interaction between plasma-wave and particles. By using the energy method to derive a priori bounds and an approximation argument for the construction of solutions, we obtain local existence and uniqueness results for the magnetic Zakharov system in the case of $d=2,3$.
\end{abstract}

AMS Subject Classification: 76B03, 35Q35

Key Words: Zakharov system, magnetic Zakharov system, local wellposedness

\section{Introduction and the main results}

In this paper we study the Cauchy problem for the magnetic Zakharov system

$$
\left\{\begin{array}{l}
i E_{t}+\nabla(\nabla \cdot E)-\alpha \nabla \times(\nabla \times E)-n E+i E \times B=0, \\
n_{t t}-\Delta n=\Delta|E|^{2}, \\
B_{t t}+\Delta^{2} B-\Delta B=-i \Delta^{2}(E \times \bar{E})
\end{array}\right.
$$

with initial data

$$
E(0, x)=E_{0}, \quad\left(n(0, x), n_{t}(0, x)\right)=\left(n_{0}, n_{1}\right),\left(B(0, x), B_{t}(0, x)\right)=\left(B_{0}, B_{1}\right),
$$

where $\alpha \geq 1$ is a constant, $x \in \mathbb{R}^{d}, d=2$, 3. The function $E: \mathbb{R} \oplus \mathbb{R}^{d} \rightarrow \mathbb{C}^{3}$ is the slowly varying amplitude of the high-frequency electric field, and the function $n: \mathbb{R} \oplus \mathbb{R}^{d} \rightarrow \mathbb{R}$ denotes the fluctuation of the ion-density from its equilibrium, and $B: \mathbb{R} \oplus \mathbb{R}^{d} \rightarrow \mathbb{R}^{3}$ is the self-generated magnetic. $\bar{E}$ denotes the conjugate complex of $E$, and the notation

*Corresponding author: zjj_math@yahoo.com.cn 
$\times$ appearing in (1.1) means the cross product for $\mathbb{R}^{3}$ or $\mathbb{C}^{3}$ valued vectors. If the space dimension $d=2, E$ and $B$ are always taken as the form $E(t, x)=\left(E_{1}(t, x), E_{2}(t, x), 0\right)$, $B(t, x)=\left(0,0, B_{3}(t, x)\right), x \in \mathbb{R}^{2}$.

Omitting the magnetic field $B$, then the system (1.1)-(1.2) reduces to the standard Zakharov system (taking $\alpha=1$ )

$$
\left\{\begin{array}{l}
i E_{t}+\Delta E=n E, \\
n_{t t}-\Delta n=\Delta|E|^{2}, \\
E(0, x)=E_{0}, n(0, x)=n_{0}, n_{t}(0, x)=n_{1} .
\end{array}\right.
$$

This system has been studied by many mathematicians in the past decades. For the Zakharov system (1.3), local existence and uniqueness of smooth solution $(E, n) \in$ $L^{\infty}\left(0, T ; H^{m} \oplus H^{m-1}\right)$ with $m \geq 3$ integer were first obtained by C. Sulem and P. L. Sulem [17, in which they also proved the solution is global in time in one spatial dimension. We also refer to [8] for the results of classical solution in one space dimensional case. In [1], H. Added and S. Added proved the smooth solution can be extended globally in time when $\left\|E_{0}\right\|_{L^{2}}$ is small in the case $d=2$. Local well-posedness in $H^{2} \oplus H^{1} \oplus L^{2}$ was shown by T. Ozawa and Y. Tsutsumi in [15]. J. Bourgain and J. Colliander [3] obtained local wellposed results in the energy norm $\left(E_{0}, n_{0}, n_{1}\right) \in H^{1} \oplus L^{2} \oplus \dot{H}^{-1}$ and showed the solution is global under small assumption on $E_{0}$ in $d=2$, 3. Furthermore, J. Ginibre, Y. Tsutsumi and G. Velo [9] established local well-posedness theory in lower regularity Sobolev spaces. For more well-posedness results for the Zakharov system (1.3), we refer to $[4,5,11,16$, and the references therein.

However, the system (1.3) ignores the effect of the magnetic filed which is generated in the laser plasma. In fact, it is meaningful to consider the self-generated magnetic field in the Zakharov system from physical viewpoint, e.g. we can study whether the magnetic field can promote the formation of soliton in three dimensions or whether it can affect the collapse process of wave packet in plasma. The magnetic $B$ has has different expressions in different plasmas. In a cold plasma, the spontaneous of a magnetic filed is given by

$$
\Delta B-i \eta \nabla \times(\nabla \times(E \times \bar{E}))+\beta B=0, \beta \leq 0, \eta>0
$$

while in a hot plasma, the magnetic filed satisfies

$$
\Delta B-i \eta \nabla \times(\nabla \times(E \times \bar{E}))-\gamma \frac{\partial}{\partial t} \int_{\mathbb{R}^{3}} \frac{B(t, y)}{|x-y|^{2}} d y=0, \eta, \gamma>0 .
$$

One can see [13] for the derivation of the above magnetic equation. In [14], C. Laurey studied the existence and uniqueness of the solution for the Zakharov system with the magnetic given by (1.4) or (1.5). Starting from Vlasov-Maxwell equations, X. He [10] first derived the magnetic system (1.1), for which describes the pondermotive force and magnetic field generation effects resulting from the non-linear interaction between plasmawave and particles. Hence, in the present paper, we are devoted to studying the Cauchy problem of the magnetic Zakharov system (1.1)-(1.2).

To obtain local well-posedness of the the magnetic Zakharov system (1.1)-(1.2), we use the energy method together with communicator estimate to derive a priori bounds and an approximation argument for the construction of solutions. After obtaining the 
uniform bounds for the approximating solutions, we prove strong convergence of these solutions, then we can get the well-posedness results. Now we state our main results.

Theorem 1.1. Assume that $s>\frac{d}{2}$, and let $D_{R}(0)$ be the set of $\left(E_{0}, n_{0}, n_{1}, B_{0}, B_{1}\right) \in$ $H^{s+1} \oplus H^{s} \oplus\left(H^{s-1} \cap \dot{H}^{-1}\right) \oplus\left(H^{s} \cap \dot{H}^{-1}\right) \oplus\left(H^{s-2} \cap \dot{H}^{-2}\right)$ such that

$$
\left\|E_{0}\right\|_{H^{s+1}}+\left\|n_{0}\right\|_{H^{s}}+\left\|n_{1}\right\|_{H^{s-1} \cap \dot{H}^{-1}}+\left\|B_{0}\right\|_{H^{s} \cap \dot{H}^{-1}}+\left\|B_{1}\right\|_{H^{s-2} \cap \dot{H}^{-2}} \leq R .
$$

Then for all $R>0$, there exists $T_{\max }=T_{\max }(R)>0$ such that for all $\left(E_{0}, n_{0}, n_{1}, B_{0}, B_{1}\right) \in D_{R}(0)$ the magnetic Zakharov system (1.1) has a unique solution $(E, n, B)$ with

$$
\begin{gathered}
(E, n, B) \in C\left(\left[0, T_{\max }\right) ; H^{s+1} \oplus H^{s} \oplus\left(H^{s} \cap \dot{H}^{-1}\right)\right), \\
\left(E_{t}, n_{t}, B_{t}\right) \in C\left(\left[0, T_{\max }\right) ; H^{s-1} \oplus\left(H^{s-1} \cap \dot{H}^{-1}\right) \oplus\left(H^{s-2} \cap \dot{H}^{-2}\right)\right) .
\end{gathered}
$$

Note that the above theorem needs the additional condition $n_{1} \in \dot{H}^{-1}, B_{0} \in \dot{H}^{-1}$ and $B_{1} \in \dot{H}^{-2}$. Since $\mathcal{S}\left(\mathbb{R}^{2}\right) \not \subset \dot{H}^{-1}\left(\mathbb{R}^{2}\right)$ and $\mathcal{S}\left(\mathbb{R}^{3}\right) \not \subset \dot{H}^{-2}\left(\mathbb{R}^{3}\right)$, the additional assumption on $n_{1}, B_{0}, B_{1}$ seems unnatural. In fact, inspired by [7], this condition can be removed by splitting the initial data into low frequency part and high frequency part. Namely, we have the following result concerning the Cauchy problem for the magnetic Zakharov system.

Theorem 1.2. Assume $s>\frac{d}{2}$, and let $\tilde{D}_{R}(0)$ be the set of $\left(E_{0}, n_{0}, n_{1}, B_{0}, B_{1}\right) \in H^{s+1} \oplus$ $H^{s} \oplus H^{s-1} \oplus H^{s} \oplus H^{s-2}$ such that

$$
\left\|E_{0}\right\|_{H^{s+1}}+\left\|n_{0}\right\|_{H^{s}}+\left\|n_{1}\right\|_{H^{s-1}}+\left\|B_{0}\right\|_{H^{s}}+\left\|B_{1}\right\|_{H^{s-2}} \leq R .
$$

Then for all $R>0$, there exists $T_{\max }=T_{\max }(R)>0$ such that for all $\left(E_{0}, n_{0}, n_{1}, B_{0}, B_{1}\right) \in \tilde{D}_{R}(0)$ the magnetic Zakharov system (1.1) has a unique solution $(E, n, B)$ with

$$
\begin{aligned}
& (E, n, B) \in C\left(\left[0, T_{\max }\right) ; H^{s+1} \oplus H^{s} \oplus H^{s}\right), \\
& \left(E_{t}, n_{t}, B_{t}\right) \in C\left(\left[0, T_{\max }\right) ; H^{s-1} \oplus H^{s-1} \oplus H^{s-2}\right) .
\end{aligned}
$$

Throughout the paper, the square root of the Laplacian $(-\Delta)^{\frac{1}{2}}$ will be denoted by $\Lambda$ and obviously

$$
\mathcal{F}(\Lambda f)=|\xi| \hat{f}
$$

We denote the inner product of $f$ and $g$ by $(f, g):=\int_{\mathbb{R}^{d}} f(x) \cdot \bar{g}(x) d x$. We define, for $s \in \mathbb{R}$ and $1 \leq p \leq \infty$, the inhomogeneous Sobolev space $H^{s, p}\left(\mathbb{R}^{d}\right)$ or simply $H^{s, p}$ of tempered distribution $f$ such that

$$
\|f\|_{H^{s, p}}=\left\|(I-\Delta)^{\frac{s}{2}} f\right\|_{L^{p}}<\infty,
$$

where $(I-\Delta)^{\frac{s}{2}} f$ is defined by $(I-\Delta)^{\frac{s}{2}} f=\mathcal{F}^{-1}\left(\left(1+|\xi|^{2}\right)^{\frac{s}{2}} \hat{f}\right)$. If $p=2$, we write $H^{s}$ instead of $H^{s, 2}$ for short, and by Plancherel's theorem $\|f\|_{H^{s}}=\left\|\left(1+|\xi|^{2}\right)^{\frac{s}{2}} \hat{f}\right\|_{L^{2}}$. For $s \in \mathbb{R}$, one can define the homogeneous Sobolev space $\dot{H}^{s, p}$ or $\dot{H}^{s}\left(=\dot{H}^{s, 2}\right)$ by

$$
\|f\|_{\dot{H}^{s, p}}=\left\|\Lambda^{s} f\right\|_{L^{p}}<\infty .
$$


This paper is organized as follows. In the next section, we derive some conserved quantities of the system (1.1), and present the existence result of weak solutions. In Section 3, we introduce a regularized system for our magnetic Zakharov system that exists a unique smooth solution globally. We derive a priori estimates for this regularized system in Section 4 and obtain the strong convergence property of the approximating solution in Section 5. Section 6 is concerned with the proof of the main theorem.

\section{Conserved quantities and weak solutions}

As we know, conserved laws paly an important role in the analytic theory(e.g. wellposedness theory and asymptotic behavior) for nonlinear PDEs of physical origin. For the magnetic Zakharov system (1.1), we have the following conserved results.

Proposition 2.1. For sufficiently regular solutions of the system (1.1), there hold two conserved quantities:

$$
\begin{aligned}
\Phi(t):= & \|E(t)\|_{L^{2}}^{2}=\Phi(0), \\
\Psi(t):= & \|\nabla \cdot E(t)\|_{L^{2}}^{2}+\alpha\|\nabla \times E(t)\|_{L^{2}}^{2}+\frac{1}{2}\|n(t)\|_{L^{2}}^{2}+\frac{1}{2}\left\|\Lambda^{-1} n_{t}(t)\right\|_{L^{2}}^{2} \\
& +\frac{1}{2}\left\|\Lambda^{-2} B_{t}(t)\right\|_{L^{2}}^{2}+\frac{1}{2}\|B(t)\|_{L^{2}}^{2}+\frac{1}{2}\left\|\Lambda^{-1} B(t)\right\|_{L^{2}}^{2} \\
& +\int_{\mathbb{R}^{d}} n(t)|E(t)|^{2} d x+i \int_{\mathbb{R}^{d}}(E(t) \times \overline{E(t)}) \cdot B(t) d x \\
& =\Psi(0) .
\end{aligned}
$$

Proof. Multiplying the first equation of (1.1) by $\bar{E}$, then integrating the imaginary part over $\mathbb{R}^{d}$, and noticing that

$$
\begin{aligned}
& 2 \operatorname{Im}\left(i E_{t} \cdot \bar{E}\right)=|E|_{t}^{2},(\nabla(\nabla \cdot E), E)=-\|\nabla \cdot E\|_{L^{2}}^{2}, \quad(n E, E)=\left\|n|E|^{2}\right\|_{L^{1}}, \\
& (\nabla \times(\nabla \times E), E)=\|\nabla \times E\|_{L^{2}}^{2}, i(E \times B) \cdot \bar{E}=i(\bar{E} \times E) \cdot B
\end{aligned}
$$

and $E \times \bar{E}$ is purely imaginary, we then obtain

$$
\frac{1}{2} \frac{d}{d t}\|E(t)\|_{L^{2}}^{2}=0
$$

from which (2.1) follows.

Now multiplying the first equation of (1.1) by $-\overline{E_{t}}$ and integrating the real part, then we have

$$
\frac{1}{2} \frac{d}{d t}\left(\|\nabla \cdot E\|_{L^{2}}^{2}+\alpha\|\nabla \times E\|_{L^{2}}^{2}\right)+\frac{1}{2} \int_{\mathbb{R}^{d}} n|E|_{t}^{2} d x-\operatorname{Re} i \int_{\mathbb{R}^{d}}(E \times B) \cdot \overline{E_{t}} d x=0 .
$$

We take inner product of the second equation of (1.1) with $\Lambda^{-2} n_{t}$ and obtain

$$
\frac{1}{2} \frac{d}{d t}\left(\left\|\Lambda^{-1} n_{t}\right\|_{L^{2}}^{2}+\|n\|_{L^{2}}^{2}\right)+\int_{\mathbb{R}^{d}} n_{t}|E|^{2} d x=0 .
$$


Similarly, if one takes inner product of the third equation of (1.1) with $\Lambda^{-4} B_{t}$, then one has

$$
\frac{1}{2} \frac{d}{d t}\left(\left\|\Lambda^{-2} B_{t}\right\|_{L^{2}}^{2}+\|B\|_{L^{2}}^{2}+\left\|\Lambda^{-1} B\right\|_{L^{2}}^{2}\right)+i \int_{\mathbb{R}^{d}}(E \times \bar{E}) \cdot B_{t} d x=0 .
$$

Since $(E \times \bar{E})_{t}=2 i \operatorname{Im}\left(E \times \overline{E_{t}}\right)$, we then have

$$
\begin{aligned}
-\operatorname{Re} i \int_{\mathbb{R}^{d}}(E \times B) \cdot \overline{E_{t}} d x & =-\operatorname{Im} \int_{\mathbb{R}^{d}}\left(E \times \overline{E_{t}}\right) \cdot B d x \\
& =\frac{i}{2} \int_{\mathbb{R}^{d}}(E \times \bar{E})_{t} \cdot B d x .
\end{aligned}
$$

Combining the equalities (2.3)-(2.6), we then get

$$
\frac{d}{d t} \Psi(t)=0
$$

which implies that $\Psi(t)=\Psi(0)$.

The conserved quantities (2.1)-(2.2) are the mail tool in establishing the global existence of weak solutions for the system (1.1)-(1.2). Before doing so, we first give the following elementary lemma.

Lemma 2.1. Assume $f(t)$ is a nonnegative continuous function in $\mathbb{R}^{+}$, and satisfies

$$
f(t) \leq a+b f^{\kappa}(t), a, b>0, \kappa>1 .
$$

If $a^{\kappa-1} b<\frac{(\kappa-1)^{\kappa-1}}{\kappa^{\kappa}}$ and $f(0) \leq a$, then $f(t)$ is bounded in $\mathbb{R}^{+}$.

Proof. Let $g(x)=a+b x^{\kappa}-x, x \geq 0$. It is easy to see that the function $g$ has a unique critical point $x_{0}=\left(\frac{1}{b \kappa}\right)^{\frac{1}{\kappa-1}}$. Hence, the condition $a^{\kappa-1} b<\frac{(\kappa-1)^{\kappa-1}}{\kappa^{\kappa}}$ ensures $f\left(x_{0}\right)<0$ which implies that there exist two points $x_{1}<x_{2}$ such that $g\left(x_{1}\right)=g\left(x_{2}\right)=0$. So if $g(x) \geq 0$, then either $x \geq x_{2}$ or $0 \leq x \leq x_{1}$. Now set $x=f(t)$, since $a<x_{1}$ and $f(t)$ is continuous, then the another condition $f(0) \leq a$ ensures $f(t) \leq x_{1}$ for all $t \geq 0$. Lemma 2.1 then follows.

Lemma 2.2. Let $(E, n, B)$ be a sufficiently regular solution to the magnetic Zakharov system (1.1)-(1.2) with $\left\|E_{0}\right\|_{L^{2}}$ small $(d=2)$ or $\left\|E_{0}\right\|_{H^{1}}$ small $(d=3)$, more precisely,

$$
\begin{aligned}
& 2\left\|E_{0}\right\|_{L^{2}}^{2}<\|Q\|_{L^{2}}^{2}, \text { if } d=2, \\
& \left\|E_{0}\right\|_{L^{2}}^{2}<\frac{1}{27 K^{8}(3)|\Psi(0)|},\left\|\nabla E_{0}\right\|_{L^{2}}^{2} \leq|\Psi(0)|, \text { if } d=3,
\end{aligned}
$$

where $Q=Q(x)$ is the ground state solution of

$$
\Delta Q-Q+Q^{3}=0, x \in \mathbb{R}^{2}
$$

Then we have

$$
\|E\|_{H^{1}}^{2}+\|n\|_{L^{2}}^{2}+\left\|n_{t}\right\|_{\dot{H}^{-1}}^{2}+\|B\|_{L^{2} \cap \dot{H}^{-1}}^{2}+\left\|B_{t}\right\|_{\dot{H}^{-2}}^{2} \leq C
$$

here the constant $C$ depends on $\left\|E_{0}\right\|_{H^{1}},\left\|n_{0}\right\|_{L^{2}},\left\|n_{1}\right\|_{\dot{H}^{-1}},\left\|B_{0}\right\|_{L^{2} \cap \dot{H}^{-1}},\left\|B_{1}\right\|_{\dot{H}^{-2}}$. 
Proof. By Cauchy-Schwarz inequality, we have

$$
\left.\left|\int_{\mathbb{R}^{d}} n\right| E\right|^{2} d x \mid \leq \epsilon\|n\|_{L^{2}}^{2}+\frac{1}{4 \epsilon}\|E\|_{L^{4}}^{4} \leq \epsilon\|n\|_{L^{2}}^{2}+\frac{1}{4 \epsilon} K^{4}(d)\|E\|_{L^{2}}^{4-d}\|\nabla E\|_{L^{2}}^{d}
$$

for all $0<\epsilon<\frac{1}{2}$, where we have used the following Sobolev best constant inequality (see [19])

$$
\|f\|_{L^{4}\left(\mathbb{R}^{d}\right)}^{4} \leq K^{4}(d)\|f\|_{L^{2}\left(\mathbb{R}^{d}\right)}^{4-d}\|\nabla f\|_{L^{2}\left(\mathbb{R}^{d}\right)}^{d},
$$

and $K^{4}(d)=\frac{2}{\|Q\|_{L^{2}}^{2}}, Q$ is the ground state solution of

$$
\frac{d}{2} \Delta Q-\left(2-\frac{d}{2}\right) Q+Q^{3}=0
$$

Similarly, we can obtain

$$
\left|i \int_{\mathbb{R}^{d}}(E(t) \times \overline{E(t)}) \cdot B(t) d x\right| \leq \epsilon\|B\|_{L^{2}}^{2}+\frac{1}{4 \epsilon} K^{4}(d)\|E\|_{L^{2}}^{4-d}\|\nabla E\|_{L^{2}}^{d}
$$

for all $0<\epsilon<\frac{1}{2}$.

Due to the fact $\|\nabla \cdot E(t)\|_{L^{2}}^{2}+\|\nabla \times E(t)\|_{L^{2}}^{2}=\|\nabla E\|_{L^{2}}^{2}$, we deduce from (2.9), (2.10) and Proposition 2.1 that if $d=2$

$$
\begin{aligned}
& \|\nabla E\|_{L^{2}}^{2}+\left(\frac{1}{2}-\epsilon\right)\|n\|_{L^{2}}^{2}+\left(\frac{1}{2}-\epsilon\right)\|B\|_{L^{2}}^{2}+\frac{1}{2}\|B\|_{\dot{H}^{-1}}^{2}+\frac{1}{2}\left\|n_{t}\right\|_{\dot{H}^{-1}}^{2}+\frac{1}{2}\left\|B_{t}\right\|_{\dot{H}^{-2}}^{2} \\
& \quad \leq|\Psi(0)|+\frac{1}{2 \epsilon} K^{4}(2)\left\|E_{0}\right\|_{L^{2}}^{2}\|\nabla E\|_{L^{2}}^{2} \\
& \quad=|\Psi(0)|+\frac{1}{\epsilon} \frac{\left\|E_{0}\right\|_{L^{2}}^{2}}{\|Q\|_{L^{2}}^{2}}\|\nabla E\|_{L^{2}}^{2} .
\end{aligned}
$$

If $2\left\|E_{0}\right\|_{L^{2}}^{2}<\|Q\|_{L^{2}}^{2}$, then we can choose $\epsilon$ very close to $\frac{1}{2}$ such that $\left\|E_{0}\right\|_{L^{2}}^{2}<\epsilon\|Q\|_{L^{2}}^{2}$, thus (2.8) follows in the case $d=2$.

If $d=3$, we can $\operatorname{obtain}\left(\epsilon=\frac{1}{4}\right)$

$$
\begin{aligned}
& \|\nabla E\|_{L^{2}}^{2}+\frac{1}{4}\|n\|_{L^{2}}^{2}+\frac{1}{4}\|B\|_{L^{2}}^{2}+\frac{1}{2}\|B\|_{\dot{H}^{-1}}^{2}+\frac{1}{2}\left\|n_{t}\right\|_{\dot{H}^{-1}}^{2}+\frac{1}{2}\left\|B_{t}\right\|_{\dot{H}^{-2}}^{2} \\
& \leq|\Psi(0)|+2 K^{4}(3)\left\|E_{0}\right\|_{L^{2}}\|\nabla E\|_{L^{2}}^{3} .
\end{aligned}
$$

If we take $f(t)=\|\nabla E\|_{L^{2}}^{2}, a=|\Psi(0)|, b=2 K^{4}(3)\left\|E_{0}\right\|_{L^{2}}, \kappa=\frac{3}{2}$, then Lemma 2.1 gives $\|\nabla E(t)\|_{L^{2}}^{2} \leq C$ for all $t \geq 0$. Hence, (2.8) is obtained from (2.11).

So an immediate application of the conservation laws (2.1)-(2.2) is to establish the existence of weak solutions to the magnetic Zakharov system (1.1).

Theorem 2.1. If $E_{0} \in H^{1},\left(n_{0}, n_{1}\right) \in L^{2} \oplus \dot{H}^{-1},\left(B_{0}, B_{1}\right) \in\left(L^{2} \cap \dot{H}^{-1}\right) \oplus \dot{H}^{-2}$, and the initial data satisfying (2.7), then there exists a weak solution $(E, n, B)$ for the system (1.1) -(1.2) in the distributional sense such that

$E \in L^{\infty}\left(\mathbb{R}^{+} ; H^{1}\right),\left(n, n_{t}\right) \in L^{\infty}\left(\mathbb{R}^{+} ; L^{2} \oplus \dot{H}^{-1}\right),\left(B, B_{t}\right) \in L^{\infty}\left(\mathbb{R}^{+} ;\left(L^{2} \cap \dot{H}^{-1}\right) \oplus \dot{H}^{-2}\right)$.

Using the prior estimate (2.8), Theorem 2.1 can be proved by applying Galerkin method and compactness argument, since this procedure is standard, the proof of Theorem 2.1 is omitted here. 


\section{Regularization for the original system}

In this section, we introduce a regularized system for our original system (1.1). Now consider the following system $(0<\epsilon<1)$

$$
\begin{aligned}
& i E_{t}^{\epsilon}+i \epsilon^{2} \Delta^{2} E_{t}^{\epsilon}+\nabla\left(\nabla \cdot E^{\epsilon}\right)-\alpha \nabla \times\left(\nabla \times E^{\epsilon}\right)-n^{\epsilon} E^{\epsilon}+i E^{\epsilon} \times B^{\epsilon}=0, \\
& n_{t t}^{\epsilon}-\Delta n^{\epsilon}=\Delta\left|E^{\epsilon}\right|^{2}, \\
& B_{t t}^{\epsilon}+\Delta^{2} B^{\epsilon}-\Delta B^{\epsilon}=-i \Delta^{2}\left(E^{\epsilon} \times \overline{E^{\epsilon}}\right)
\end{aligned}
$$

with smooth initial data

$$
E^{\epsilon}(0)=E_{0}^{\epsilon}, n^{\epsilon}(0)=n_{0}^{\epsilon}, n_{t}^{\epsilon}(0)=n_{1}^{\epsilon}, B^{\epsilon}(0)=B_{0}^{\epsilon}, B_{t}^{\epsilon}(0)=B_{1}^{\epsilon} .
$$

With the same argument as Proposition 2.1, we can obtain some conservation results for this regularized system.

Proposition 3.1. Assume $\left(E^{\epsilon}, n^{\epsilon}, B^{\epsilon}\right)$ is a sufficient regular solution for the system (3.1a) - (3.1d), then we have

$$
\begin{aligned}
\Phi^{\epsilon}(t):= & \left\|E^{\epsilon}(t)\right\|_{L^{2}}^{2}+\epsilon^{2}\left\|\Delta E^{\epsilon}(t)\right\|_{L^{2}}^{2}=\Phi^{\epsilon}(0) \\
\Psi^{\epsilon}(t):= & \left\|\nabla \cdot E^{\epsilon}(t)\right\|_{L^{2}}^{2}+\alpha\left\|\nabla \times E^{\epsilon}(t)\right\|_{L^{2}}^{2}+\frac{1}{2}\left\|n^{\epsilon}(t)\right\|_{L^{2}}^{2}+\frac{1}{2}\left\|\Lambda^{-1} n_{t}^{\epsilon}(t)\right\|_{L^{2}}^{2} \\
& +\frac{1}{2}\left\|\Lambda^{-2} B_{t}^{\epsilon}(t)\right\|_{L^{2}}^{2}+\frac{1}{2}\left\|B^{\epsilon}(t)\right\|_{L^{2}}^{2}+\frac{1}{2}\left\|\Lambda^{-1} B^{\epsilon}(t)\right\|_{L^{2}}^{2} \\
& +\int_{\mathbb{R}^{d}} n^{\epsilon}(t)\left|E^{\epsilon}(t)\right|^{2} d x+i \int_{\mathbb{R}^{d}}\left(E^{\epsilon}(t) \times \overline{E^{\epsilon}(t)}\right) \cdot B^{\epsilon}(t) d x \\
& =\Psi^{\epsilon}(0) .
\end{aligned}
$$

Let $\mathcal{L}=\left(I+\epsilon^{2} \Delta^{2}\right)^{-1}$, and let $\mathcal{A}$ be the linear operator defined by

$$
\mathcal{A} E=-\nabla(\nabla \cdot E)+\alpha \nabla \times(\nabla \times E)
$$

since the operator $\mathcal{L} \mathcal{A}$ is self-adjoint, then the linear equation

$$
i E_{t}=\mathcal{L} \mathcal{A} E, E(0)=E_{0}
$$

generates a unitary group $U(t)$ in $H^{r}\left(\mathbb{R}^{d}\right)$. Therefore, we can transform the regularized system (3.1a)-(3.1d) into the following integral equation

$$
E^{\epsilon}(t)=U(t) E_{0}^{\epsilon}+\int_{0}^{t} U(t-\tau) f\left(E^{\epsilon}(\tau)\right) d \tau,
$$

where $f\left(E^{\epsilon}(t)\right)=-i \mathcal{L}\left(n^{\epsilon} E^{\epsilon}\right)-\mathcal{L}\left(E^{\epsilon} \times B^{\epsilon}\right)$, and $n^{\epsilon}=n^{\epsilon}\left(E^{\epsilon}\right), B^{\epsilon}=B^{\epsilon}\left(E^{\epsilon}\right)$ is the solution of equation (3.1b), (3.1c) respectively. Indeed, we can express $n^{\epsilon}\left(E^{\epsilon}\right), B^{\epsilon}\left(E^{\epsilon}\right)$ by

$$
\begin{aligned}
n^{\epsilon}\left(E^{\epsilon}\right)= & \cos \left((-\Delta)^{1 / 2} t\right) n_{0}^{\epsilon}+\frac{\sin \left((-\Delta)^{1 / 2} t\right)}{(-\Delta)^{1 / 2}} n_{1}^{\epsilon} \\
& +\int_{0}^{t} \frac{\sin \left((-\Delta)^{1 / 2}(t-\tau)\right)}{(-\Delta)^{1 / 2}} \Delta\left|E^{\epsilon}(\tau)\right|^{2} d \tau
\end{aligned}
$$


and

$$
\begin{aligned}
B^{\epsilon}\left(E^{\epsilon}\right)= & \cos \left((-\Delta)^{1 / 2}\langle\nabla\rangle t\right) B_{0}^{\epsilon}+\frac{\sin \left((-\Delta)^{1 / 2}\langle\nabla\rangle t\right)}{(-\Delta)^{1 / 2}\langle\nabla\rangle} B_{1}^{\epsilon} \\
& -i \int_{0}^{t} \frac{\sin \left((-\Delta)^{1 / 2}\langle\nabla\rangle(t-\tau)\right)}{(-\Delta)^{1 / 2}\langle\nabla\rangle} \Delta^{2}\left(E^{\epsilon}(\tau) \times \overline{E^{\epsilon}(\tau)}\right) d \tau
\end{aligned}
$$

here $\langle\nabla\rangle$ is the Fourier multiplier with symbol $\langle\xi\rangle=\left(1+|\xi|^{2}\right)^{1 / 2}$.

The main result in this section is the following global existence of smooth solution for the regularized system (3.1a)-(3.1d).

Theorem 3.1. Given $\epsilon \in(0,1)$, and suppose that $E_{0}^{\epsilon} \in H^{r+1},\left(n_{0}^{\epsilon}, n_{1}^{\epsilon}\right) \in H^{r} \oplus\left(H^{r-1} \cap\right.$ $\left.\left.\dot{H}^{-1}\right),\left(B_{0}^{\epsilon}, B_{1}^{\epsilon}\right) \in\left(H^{r-1} \cap \dot{H}^{-1}\right) \oplus\left(H^{r-3} \cap \dot{H}^{-2}\right)\right), r$ is large enough $\left(\right.$ e.g. $\left.r>\frac{d}{2}+s+100\right)$, then there exists a unique smooth solution $\left(E^{\epsilon}, n^{\epsilon}, B^{\epsilon}\right)$ for the regularized system (3.1a)(3.1d) such that

$$
\left(E^{\epsilon}, n^{\epsilon}, B^{\epsilon}\right) \in C\left(\mathbb{R}^{+} ; H^{r+1} \oplus H^{r} \oplus H^{r-1}\right) .
$$

In order to prove Theorem 3.1, we first state the following calculus inequality which will be used many times in this paper.

Lemma 3.1. Assume that $s>0$ and $p \in(1,+\infty)$. If $f, g \in \mathcal{S}\left(\mathbb{R}^{d}\right)$, the Schwartz class, then

$$
\left\|\Lambda^{s}(f g)\right\|_{L^{p}} \leq C\left(\|f\|_{L^{p_{1}}}\|g\|_{\dot{H}^{s, p_{2}}}+\|f\|_{\dot{H}^{s, p_{3}}}\|g\|_{L^{p_{4}}}\right)
$$

and

$$
\left\|\Lambda^{s}(f g)-f\left(\Lambda^{s} g\right)\right\|_{L^{p}} \leq C\|\nabla f\|_{L^{p_{1}}}\|g\|_{\dot{H}^{s-1, p_{2}}}+\|f\|_{\dot{H}^{s, p_{3}}}\|g\|_{L^{p_{4}}}
$$

with $p_{2}, p_{3} \in(1,+\infty)$ such that

$$
\frac{1}{p}=\frac{1}{p_{1}}+\frac{1}{p_{2}}=\frac{1}{p_{3}}+\frac{1}{p_{4}}
$$

For a proof of this lemma, we refer to [6, 12].

Proof of Theorem 3.1. By contraction argument, we first show that equation (3.4) has a unique solution locally, then we extend this solution globally in time based on some uniform estimates.

Denote $M=\left\|E_{0}^{\epsilon}\right\|_{H^{r+1}}+\left\|n_{0}^{\epsilon}\right\|_{H^{r}}+\left\|n_{1}^{\epsilon}\right\|_{H^{r-1}}+\left\|B_{0}^{\epsilon}\right\|_{H^{r-1}}+\left\|B_{1}^{\epsilon}\right\|_{H^{r-3}}$. Let $0<T \leq 1$ be determined later, and set $X=C\left([0, T] ; H^{r+1}\right), X_{M}=\left\{E \in X ;\|E\|_{X} \leq 2 M\right\}$. Now we define the map $\mathcal{T}$ acting on $X$ by

$$
\mathcal{T}\left(E^{\epsilon}\right)=U(t) E_{0}^{\epsilon}+\int_{0}^{t} U(t-\tau) f\left(E^{\epsilon}(\tau)\right) d \tau, \forall E^{\epsilon} \in X .
$$

Our aim is to show that $\mathcal{T}$ has unique fixed point on $X$ if $T$ is small enough.

Given $E^{\epsilon} \in X$, we obtain from (3.5) that

$$
\left\|n^{\epsilon}(t)\right\|_{H^{r}} \leq(1+T)\left(\left\|n_{0}^{\epsilon}\right\|_{H^{r}}+\left\|n_{1}^{\epsilon}\right\|_{H^{r-1}}\right)+T\left\|E^{\epsilon}\right\|_{X}^{2}, \forall t \in[0, T] .
$$


Similarly, from (3.6) we have

$$
\left\|B^{\epsilon}(t)\right\|_{H^{r-1}} \leq(1+T)\left(\left\|B_{0}^{\epsilon}\right\|_{H^{r-1}}+\left\|n_{1}^{\epsilon}\right\|_{H^{r-1}}\right)+T\left\|E^{\epsilon}\right\|_{X}^{2}, \forall t \in[0, T] .
$$

Note also that $\mathcal{L}$ is a bounded linear operator from $H^{k-4}$ to $H^{k}$, namely, there exists $K>0$ not depending on $k$ such that

$$
\|\mathcal{L} f\|_{H^{k}} \leq K\|f\|_{H^{k-4}}, \forall f \in H^{k-4} .
$$

This fact together with (3.10)-(3.11) yield

$$
\begin{aligned}
\left\|f\left(E^{\epsilon}\right)\right\|_{X} & \leq K\left(\left\|n^{\epsilon}\left(E^{\epsilon}\right) E^{\epsilon}\right\|_{C\left([0, T] ; H^{r-3}\right)}+\left\|E^{\epsilon} \times B^{\epsilon}\left(E^{\epsilon}\right)\right\|_{C\left([0, T] ; H^{r-3}\right)}\right) \\
& \leq K \sup _{t \in[0, T]}\left(\left\|n^{\epsilon}\left(E^{\epsilon}\right)\right\|_{H^{r}}\left\|E^{\epsilon}\right\|_{H^{r+1}}+\left\|B^{\epsilon}\left(E^{\epsilon}\right)\right\|_{H^{r-1}}\left\|E^{\epsilon}\right\|_{H^{r+1}}\right) \\
& \leq 4 K M^{2}(1+4 M), \text { if } E^{\epsilon} \in X_{M},
\end{aligned}
$$

then we have

$$
\begin{aligned}
\left\|\mathcal{T}\left(E^{\epsilon}\right)\right\|_{X} & \leq\left\|E_{0}^{\epsilon}\right\|_{H^{r+1}}+\int_{0}^{T}\left\|f\left(E^{\epsilon}\right)\right\|_{X} d \tau \\
& \leq M+4 K M^{2}(1+4 M) T
\end{aligned}
$$

Hence we see that if $T \leq \min \left\{\frac{1}{4 K M(1+4 M)}, 1\right\}=: T_{1}$, then $\mathcal{T}$ maps $X_{M}$ into itself.

From the expression (3.5) and (3.6), we also have

$$
\begin{aligned}
\left\|n\left(E_{1}^{\epsilon}\right)(t)-n\left(E_{2}^{\epsilon}\right)(t)\right\|_{H^{r}} & \leq T\left(\left\|E_{1}^{\epsilon}\right\|_{X}+\left\|E_{2}^{\epsilon}\right\|_{X}\right)\left\|E_{1}^{\epsilon}-E_{2}^{\epsilon}\right\|_{X}, \quad \forall t \in[0, T], \\
\left\|B\left(E_{1}^{\epsilon}\right)(t)-B\left(E_{2}^{\epsilon}\right)(t)\right\|_{H^{r-1}} & \leq T\left(\left\|E_{1}^{\epsilon}\right\|_{X}+\left\|E_{2}^{\epsilon}\right\|_{X}\right)\left\|E_{1}^{\epsilon}-E_{2}^{\epsilon}\right\|_{X}, \quad \forall t \in[0, T] .
\end{aligned}
$$

Using the above two estimates and (3.10)-(3.11), we know

$$
\begin{aligned}
\left\|f\left(E_{1}^{\epsilon}\right)-f\left(E_{2}^{\epsilon}\right)\right\|_{X} \\
\leq K \sup _{t \in[0, T]}\left(\left\|n^{\epsilon}\left(E_{1}^{\epsilon}\right) E_{1}^{\epsilon}-n^{\epsilon}\left(E_{2}^{\epsilon}\right) E_{2}^{\epsilon}\right\|_{H^{r-3}}+\left\|E_{1}^{\epsilon} \times B^{\epsilon}\left(E_{1}^{\epsilon}\right)-E_{2}^{\epsilon} \times B^{\epsilon}\left(E_{2}^{\epsilon}\right)\right\|_{H^{r-3}}\right) \\
\leq K \sup _{t \in[0, T]}\left(\left\|n^{\epsilon}\left(E_{1}^{\epsilon}\right)\right\|_{H^{r}}\left\|E_{1}^{\epsilon}-E_{2}^{\epsilon}\right\|_{H^{r+1}}+\left\|n^{\epsilon}\left(E_{1}^{\epsilon}\right)-n^{\epsilon}\left(E_{2}^{\epsilon}\right)\right\|_{H^{r}}\left\|E_{2}^{\epsilon}\right\|_{H^{r+1}}\right. \\
\left.\quad+\left\|B^{\epsilon}\left(E_{1}^{\epsilon}\right)\right\|_{H^{r-1}}\left\|E_{1}^{\epsilon}-E_{2}^{\epsilon}\right\|_{H^{r+1}}+\left\|B^{\epsilon}\left(E_{1}^{\epsilon}\right)-B^{\epsilon}\left(E_{2}^{\epsilon}\right)\right\|_{H^{r-1}}\left\|E_{2}^{\epsilon}\right\|_{H^{r+1}}\right) \\
\leq K\left(24 M^{2}+2 M\right)\left\|E_{1}^{\epsilon}-E_{2}^{\epsilon}\right\|_{X}, \text { if } E_{1}^{\epsilon}, E_{2}^{\epsilon} \in X_{M} .
\end{aligned}
$$

Therefore, there holds

$$
\left\|\mathcal{T}\left(E_{1}^{\epsilon}\right)-\mathcal{T}\left(E_{2}^{\epsilon}\right)\right\|_{X} \leq T K\left(24 M^{2}+2 M\right)\left\|E_{1}^{\epsilon}-E_{2}^{\epsilon}\right\|_{X}
$$

If we choose $T \leq \min \left\{\frac{1}{2 K\left(24 M^{2}+2 M\right)}, T_{1}\right\}$, then $\mathcal{T}$ is a contraction map on $X_{M}$. So by fixed point theorem, we know that the equation (3.9) has a unique solution $E^{\epsilon} \in$ $C\left([0, T] ; H^{r+1}\right)$, and by (3.5) $-(3.6)$, we know $n^{\epsilon} \in C\left([0, T] ; H^{r}\right), B^{\epsilon} \in C\left([0, T] ; H^{r-1}\right)$.

Assume now $T_{\max }$ is the maximal existence time of the solution $\left(E^{\epsilon}, n^{\epsilon}, B^{\epsilon}\right)$ for the regularized system (3.1a - (3.1d), hence in order to complete the proof of Theorem 3.1. 
we have to show that $T_{\max }=\infty$. To this end, it is sufficient to prove that the quantities $\left\|E^{\epsilon}\right\|_{H^{r+1}},\left\|n^{\epsilon}\right\|_{H^{r}},\left\|n_{t}^{\epsilon}\right\|_{H^{r-1}},\left\|B^{\epsilon}\right\|_{H^{r-1}},\left\|B_{t}^{\epsilon}\right\|_{H^{r-3}}$ are bounded on the time interval $\left[0, T_{\max }\right)$.

It follows from (3.2) that

$$
\left\|E^{\epsilon}\right\|_{H^{2}} \leq C\left(\Rightarrow\left\|E^{\epsilon}\right\|_{L^{\infty}} \leq C\right)
$$

which in turn gives that(by (3.5) and (3.6)

$$
\left\|n^{\epsilon}\right\|_{H^{1}} \leq C,\left\|B^{\epsilon}\right\|_{L^{2}} \leq C .
$$

Besides, one also deduces from (3.3) that

$$
\left\|n_{t}^{\epsilon}\right\|_{\dot{H}^{-1}}+\left\|B_{t}^{\epsilon}\right\|_{\dot{H}^{-2}} \leq C .
$$

We emphasize that the constant $C$ in the above estimates depends on the parameter $\epsilon$.

Now multiplying (3.1a) by $\Lambda^{2 r-2} \overline{E^{\epsilon}}$, and integrating the imaginary part, since

$$
\operatorname{Im}\left(\nabla\left(\nabla \cdot E^{\epsilon}\right)-\alpha \nabla \times\left(\nabla \times E^{\epsilon}\right), \Lambda^{2 r-2} E^{\epsilon}\right)=0,
$$

then we obtain from (3.7), (3.12)-(3.13) that

$$
\begin{aligned}
\frac{1}{2} \frac{d}{d t}\left(\left\|\Lambda^{r-1} E^{\epsilon}\right\|_{L^{2}}^{2}+\epsilon^{2}\left\|\Lambda^{r+1} E^{\epsilon}\right\|_{L^{2}}^{2}\right) \\
=\int_{\mathbb{R}^{d}} \Lambda^{r-1}\left(n^{\epsilon} E^{\epsilon}\right) \Lambda^{r-1} \overline{E^{\epsilon}} d x-i \int_{\mathbb{R}^{d}} \Lambda^{r-1}\left(E^{\epsilon} \times B^{\epsilon}\right) \Lambda^{r-1} \overline{E^{\epsilon}} d x \\
\leq C\left(\left\|n^{\epsilon}\right\|_{H^{r-1}}\left\|E^{\epsilon}\right\|_{L^{\infty}}+\left\|n^{\epsilon}\right\|_{L^{4}}\left\|E^{\epsilon}\right\|_{H^{r-1,4}}\right)\left\|\Lambda^{r-1} \overline{E^{\epsilon}}\right\|_{L^{2}} \\
\quad+C\left(\left\|B^{\epsilon}\right\|_{H^{r-1}}\left\|E^{\epsilon}\right\|_{L^{\infty}}+\left\|B^{\epsilon}\right\|_{L^{p}}\left\|E^{\epsilon}\right\|_{H^{r-1, q}}\right)\left\|\Lambda^{r-1} \overline{E^{\epsilon}}\right\|_{L^{2}} \\
\leq C\left(\left\|n^{\epsilon}\right\|_{H^{r}}+\left\|B^{\epsilon}\right\|_{H^{r-1}}+\left\|E^{\epsilon}\right\|_{H^{r+1}}\right)\left\|E^{\epsilon}\right\|_{H^{r+1}} \\
\leq C\left(\left\|n^{\epsilon}\right\|_{H^{r}}^{2}+\left\|B^{\epsilon}\right\|_{H^{r-1}}^{2}+\left\|E^{\epsilon}\right\|_{H^{r+1}}^{2}\right),
\end{aligned}
$$

where we have used the following inequality $\left(\frac{1}{p}+\frac{1}{q}=\frac{1}{2}\right)$

$$
\varlimsup_{p \rightarrow 2^{+}}\left\|B^{\epsilon}\right\|_{L^{p}}\left\|E^{\epsilon}\right\|_{H^{r-1, q}} \leq C\left\|E^{\epsilon}\right\|_{H^{r+1}} \varlimsup_{p \rightarrow 2^{+}}\left\|B^{\epsilon}\right\|_{L^{p}} \leq C\left\|E^{\epsilon}\right\|_{H^{r+1}}
$$

due to the fact $\left\|B^{\epsilon}\right\|_{L^{2}} \leq C$.

We then multiply (3.1b) by $\Lambda^{2 r-2} n_{t}^{\epsilon}$ and obtain

$$
\begin{aligned}
& \frac{1}{2} \frac{d}{d t}\left(\left\|\Lambda^{r-1} n_{t}^{\epsilon}\right\|_{L^{2}}^{2}+\left\|\Lambda^{r} n^{\epsilon}\right\|_{L^{2}}^{2}\right) \\
& \quad=-\int_{\mathbb{R}^{d}} \Lambda^{r+1}\left(E^{\epsilon} \cdot \overline{E^{\epsilon}}\right) \Lambda^{r-1} n_{t}^{\epsilon} d x \\
& \quad \leq C\left(\left\|E^{\epsilon}\right\|_{H^{r+1}}\left\|\overline{E^{\epsilon}}\right\|_{L^{\infty}}+\left\|E^{\epsilon}\right\|_{L^{\infty}}\left\|\overline{E^{\epsilon}}\right\|_{H^{r+1}}\right)\left\|\Lambda^{r-1} n_{t}^{\epsilon}\right\|_{L^{2}} \\
& \quad \leq C\left(\left\|n_{t}^{\epsilon}\right\|_{H^{r-1}}^{2}+\left\|E^{\epsilon}\right\|_{H^{r+1}}^{2}\right) .
\end{aligned}
$$


Similarly, by taking inner product of (3.1c) with $\Lambda^{2 r-6} B_{t}^{\epsilon}$, then the same argument as above leads to

$$
\begin{aligned}
& \frac{1}{2} \frac{d}{d t}\left(\left\|\Lambda^{r-3} B_{t}^{\epsilon}\right\|_{L^{2}}^{2}+\left\|\Lambda^{r-1} B^{\epsilon}\right\|_{L^{2}}^{2}+\left\|\Lambda^{r-2} B^{\epsilon}\right\|_{L^{2}}^{2}\right) \\
& \quad=\int_{\mathbb{R}^{d}} \Lambda^{r+1}\left(E^{\epsilon} \times \overline{E^{\epsilon}}\right) \Lambda^{r-3} n_{t}^{\epsilon} d x \\
& \quad \leq C\left(\left\|B_{t}^{\epsilon}\right\|_{H^{r-3}}^{2}+\left\|E^{\epsilon}\right\|_{H^{r+1}}^{2}\right) .
\end{aligned}
$$

Now summing the estimates (3.15)-(3.17), and integrating the result, we can obtain

$$
\begin{gathered}
\left\|\Lambda^{r-1} E^{\epsilon}\right\|_{L^{2}}^{2}+\epsilon^{2}\left\|\Lambda^{r+1} E^{\epsilon}\right\|_{L^{2}}^{2}+\left\|\Lambda^{r-1} n_{t}^{\epsilon}\right\|_{L^{2}}^{2}+\left\|\Lambda^{r} n^{\epsilon}\right\|_{L^{2}}^{2} \\
+\left\|\Lambda^{r-3} B_{t}^{\epsilon}\right\|_{L^{2}}^{2}+\left\|\Lambda^{r-1} B^{\epsilon}\right\|_{L^{2}}^{2}+\left\|\Lambda^{r-2} B^{\epsilon}\right\|_{L^{2}}^{2} \\
\leq C+C \int_{0}^{t}\left(\left\|n^{\epsilon}\right\|_{H^{r}}^{2}+\left\|B^{\epsilon}\right\|_{H^{r-1}}^{2}+\left\|E^{\epsilon}\right\|_{H^{r+1}}^{2}+\left\|n_{t}^{\epsilon}\right\|_{H^{r-1}}^{2}+\left\|B_{t}^{\epsilon}\right\|_{H^{r-3}}^{2}\right) d \tau,
\end{gathered}
$$

this inequality together with the fact $\left\|E^{\epsilon}\right\|_{L^{2}}^{2} \leq C$ and (3.13)-(3.14) yield

$$
\begin{aligned}
& \left\|E^{\epsilon}\right\|_{H^{r+1}}^{2}+\left\|n_{t}^{\epsilon}\right\|_{H^{r-1}}^{2}+\left\|n^{\epsilon}\right\|_{H^{r}}^{2}+\left\|B_{t}^{\epsilon}\right\|_{H^{r-3}}^{2}+\left\|B^{\epsilon}\right\|_{H^{r-1}}^{2} \\
& \quad \leq C+C \int_{0}^{t}\left(\left\|E^{\epsilon}\right\|_{H^{r+1}}^{2}+\left\|n^{\epsilon}\right\|_{H^{r}}^{2}+\left\|n_{t}^{\epsilon}\right\|_{H^{r-1}}^{2}+\left\|B^{\epsilon}\right\|_{H^{r-1}}^{2}+\left\|B_{t}^{\epsilon}\right\|_{H^{r-3}}^{2}\right) d \tau,
\end{aligned}
$$

hence by Gronwall's inequality, we get

$$
\left\|E^{\epsilon}\right\|_{H^{r+1}}^{2}+\left\|n_{t}^{\epsilon}\right\|_{H^{r-1}}^{2}+\left\|n^{\epsilon}\right\|_{H^{r}}^{2}+\left\|B_{t}^{\epsilon}\right\|_{H^{r-3}}^{2}+\left\|B^{\epsilon}\right\|_{H^{r-1}}^{2} \leq C, \forall t \in\left[0, T_{\max }\right) .
$$

The estimate (3.18) implies that the solution $\left(E^{\epsilon}, n^{\epsilon}, B^{\epsilon}\right)$ can be extended to the interval $\left[0, T_{\max }+\delta\right]$, which contradicts the maximality, hence $T_{\max }=\infty$. Therefore, the solution for regularized system (3.1a)-(3.1d) exists globally in time, and the proof of Theorem 3.1 is complete.

\section{A prior estimates}

We will approximate the solution of the magnetic Zakharov system (1.1)-(1.2) by smooth solutions for the regularized system given in Section 3. Hence, in order to get strong or weak limit of these smooth solutions in the $L_{t}^{\infty} H_{x}^{s}$ topology, one must demonstrate the approximating solutions are uniformly bounded in this energy norm. Therefore, we are devoted to establishing a prior estimates for the system (3.1a)-3.1d) in this section.

Proposition 4.1. Let $s>\frac{d}{2}, E_{0} \in H^{s+1},\left(n_{0}, n_{1}\right) \in H^{s} \oplus\left(H^{s-1} \cap \dot{H}^{-1}\right),\left(B_{0}, B_{1}\right) \in$ $\left(H^{s} \cap \dot{H}^{-1}\right) \oplus\left(H^{s-2} \cap \dot{H}^{-2}\right)$. Assume the sequence $\left\{\left(E_{0}^{\epsilon}, n_{0}^{\epsilon}, n_{1}^{\epsilon}, B_{0}^{\epsilon}, B_{1}^{\epsilon}\right)\right\}$ satisfying $E_{0}^{\epsilon} \in$ $H^{r+1},\left(n_{0}^{\epsilon}, n_{1}^{\epsilon}\right) \in H^{r} \oplus\left(H^{r-1} \cap \dot{H}^{-1}\right),\left(B_{0}^{\epsilon}, B_{1}^{\epsilon}\right) \in\left(H^{r-1} \cap \dot{H}^{-1}\right) \oplus\left(H^{r-3} \cap \dot{H}^{-2}\right)$ with $r$ large enough, and

$$
\begin{gathered}
E_{0}^{\epsilon} \rightarrow E_{0} \text { in } H^{s+1}, \\
n_{0}^{\epsilon} \rightarrow n_{0} \text { in } H^{s}, n_{1}^{\epsilon} \rightarrow n_{1} \text { in } H^{s-1} \cap \dot{H}^{-1}, \\
B_{0}^{\epsilon} \rightarrow B_{0} \text { in } H^{s} \cap \dot{H}^{-1}, B_{1}^{\epsilon} \rightarrow B_{1} \text { in } H^{s-2} \cap \dot{H}^{-2} .
\end{gathered}
$$


If $\left(E^{\epsilon}, n^{\epsilon}, B^{\epsilon}\right)$ is the smooth solution of regularized system (3.1a)-(3.1c) with the initial data $\left(E_{0}^{\epsilon}, n_{0}^{\epsilon}, n_{1}^{\epsilon}, B_{0}^{\epsilon}, B_{1}^{\epsilon}\right)$, then there exist $T>0$ and $C>0$ such that

$$
\begin{aligned}
& \left\|E^{\epsilon}\right\|_{C\left([0, T] ; H^{s+1}\right)}+\left\|n^{\epsilon}\right\|_{C\left([0, T] ; H^{s}\right)}+\left\|B^{\epsilon}\right\|_{C\left([0, T] ; H^{s} \cap \dot{H}^{-1}\right)} \leq C, \\
& \left\|E_{t}^{\epsilon}\right\|_{C\left([0, T] ; H^{s-1}\right)}+\left\|n_{t}^{\epsilon}\right\|_{C\left([0, T] ; H^{s-1} \cap \dot{H}^{-1}\right)}+\left\|B_{t}^{\epsilon}\right\|_{C\left([0, T] ; H^{s-2} \cap \dot{H}^{-2}\right)} \leq C,
\end{aligned}
$$

where $T$ and $C$ are dependent of the norm of $\left(E_{0}, n_{0}, n_{1}, B_{0}, B_{1}\right)$, but independent of $\epsilon$.

Proof. Note that the bound for the left hand side of (3.18) depends on $\epsilon$, hence, one can not use the same argument that leads to (3.18) to obtain the estimate (4.2)-(4.3). In order to derive independent of $\epsilon$, we first write (3.1a) in the following form

$$
i E_{t}^{\epsilon}+\mathcal{L} \nabla\left(\nabla \cdot E^{\epsilon}\right)-\alpha \mathcal{L} \nabla \times\left(\nabla \times E^{\epsilon}\right)-\mathcal{L}\left(n^{\epsilon} E^{\epsilon}\right)+i \mathcal{L}\left(E^{\epsilon} \times B^{\epsilon}\right)=0,
$$

where $\mathcal{L}=\left(I+\epsilon^{2} \Delta^{2}\right)^{-1}$. It is easily to see that $\mathcal{L}$ satisfies the following properties:

$$
\left\{\begin{array}{l}
(1)\|\mathcal{L} f\|_{H^{k}} \leq\|f\|_{H^{k}}, \forall k \in \mathbb{R}, \\
(2)(\mathcal{L} f, f)=\int_{\mathbb{R}^{d}}(\mathcal{L} f) \cdot \bar{f} d x \geq 0, \\
(3)(\mathcal{L} f, g)=(f, \mathcal{L} g), \\
(4) \mathcal{L} \text { commutes with Fourier multipler such as } \Lambda^{s}, \nabla \text { and so on. }
\end{array}\right.
$$

Due to these properties, the operator $\mathcal{L}$ can be easily dealt with in the following estimates.

Since (4.1) holds, we have

$$
\left\|E_{0}^{\epsilon}\right\|_{H^{s+1}}+\left\|n_{0}^{\epsilon}\right\|_{H^{s}}+\left\|n_{1}^{\epsilon}\right\|_{H^{s-1} \cap \dot{H}^{-1}}+\left\|B_{0}^{\epsilon}\right\|_{H^{s} \cap \dot{H}^{-1}}+\left\|B_{1}^{\epsilon}\right\|_{H^{s-2} \cap \dot{H}^{-2}} \leq c_{0},
$$

where the magnitude of $c_{0}$ depends only on $\left\|E_{0}\right\|_{H^{s+1}},\left\|n_{0}\right\|_{H^{s}}, \quad\left\|n_{1}\right\|_{H^{s-1} \cap \dot{H}^{-1}}$, $\left\|B_{0}\right\|_{H^{s} \cap \dot{H}^{-1}}$ and $\left\|B_{1}\right\|_{H^{s-2} \cap \dot{H}^{-2}}$.

\section{- Low order norm estimates.}

By the conserved quantities (3.2) and (3.3), there holds

$$
\begin{aligned}
& \left\|E^{\epsilon}\right\|_{H^{1}}^{2}+\left\|n^{\epsilon}\right\|_{L^{2}}^{2}+\left\|n_{t}^{\epsilon}\right\|_{\dot{H}^{-1}}^{2}+\left\|B^{\epsilon}\right\|_{L^{2}}^{2}+\left\|B^{\epsilon}\right\|_{\dot{H}^{-1}}^{2}+\left\|B_{t}^{\epsilon}\right\|_{\dot{H}^{-2}}^{2} \\
& \leq C\left(c_{0}\right)+\left.\left|\int_{\mathbb{R}^{d}} n^{\epsilon}\right| E^{\epsilon}\right|^{2} d x|+| \int_{\mathbb{R}^{d}}\left(E^{\epsilon} \times \overline{E^{\epsilon}}\right) \cdot B^{\epsilon} d x \mid .
\end{aligned}
$$

Then by Cauchy-Schwarz inequality, we have

$$
\begin{aligned}
& \left.\left|\int_{\mathbb{R}^{d}} n^{\epsilon}\right| E^{\epsilon}\right|^{2} d x \mid \leq\left\|n^{\epsilon}\right\|_{L^{2}}\left\|E^{\epsilon}\right\|_{L^{4}}^{2} \leq \frac{1}{2}\left\|n^{\epsilon}\right\|_{L^{2}}^{2}+\frac{1}{2}\left\|E^{\epsilon}\right\|_{L^{4}}^{4}, \\
& \left|\int_{\mathbb{R}^{d}}\left(E^{\epsilon} \times \overline{E^{\epsilon}}\right) \cdot B^{\epsilon} d x\right| \leq \frac{1}{2}\left\|B^{\epsilon}\right\|_{L^{2}}^{2}+\frac{1}{2}\left\|E^{\epsilon}\right\|_{L^{4}}^{4} .
\end{aligned}
$$

Since $\left|E^{\epsilon}\right|_{t}^{2}=2 \operatorname{Im}\left(i E_{t}^{\epsilon} \cdot \overline{E^{\epsilon}}\right)$, then (4.4) yields

$$
\left|E^{\epsilon}\right|_{t}^{2}=2 \operatorname{Im}\left[\left(-\mathcal{L} \nabla\left(\nabla \cdot E^{\epsilon}\right)+\alpha \mathcal{L} \nabla \times\left(\nabla \times E^{\epsilon}\right)+\mathcal{L}\left(n^{\epsilon} E^{\epsilon}\right)-i \mathcal{L}\left(E^{\epsilon} \times B^{\epsilon}\right)\right) \cdot \overline{E^{\epsilon}}\right],
$$


hence we can obtain

$$
\begin{aligned}
\frac{d}{d t} \int_{\mathbb{R}^{d}}\left|E^{\epsilon}\right|^{4} d x & =2 \int_{\mathbb{R}^{d}}\left|E^{\epsilon}\right|^{2}\left|E^{\epsilon}\right|_{t}^{2} d x \\
& \leq C\left(\left\|E^{\epsilon}\right\|_{H^{s+1}}^{4}+\left\|E^{\epsilon}\right\|_{H^{s+1}}^{4}\left(\left\|n^{\epsilon}\right\|_{H^{s}}+\left\|B^{\epsilon}\right\|_{H^{s}}\right)\right) \\
& \leq C\left(\left\|E^{\epsilon}\right\|_{H^{s+1}}^{2}+\left\|n^{\epsilon}\right\|_{H^{s}}^{2}+\left\|B^{\epsilon}\right\|_{H^{s}}^{2}+1\right)^{3}
\end{aligned}
$$

which implies that

$$
\left\|E^{\epsilon}\right\|_{L^{4}}^{4} \leq C+C \int_{0}^{t}\left(\left\|E^{\epsilon}\right\|_{H^{s+1}}^{2}+\left\|n^{\epsilon}\right\|_{H^{s}}^{2}+\left\|B^{\epsilon}\right\|_{H^{s}}^{2}+1\right)^{3} d \tau .
$$

Putting (4.7), (4.8), (4.10) together, then we have

$$
\text { LHS of (4.77) } \leq C+C \int_{0}^{t}\left(\left\|E^{\epsilon}\right\|_{H^{s+1}}^{2}+\left\|n^{\epsilon}\right\|_{H^{s}}^{2}+\left\|B^{\epsilon}\right\|_{H^{s}}^{2}+1\right)^{3} d \tau \text {. }
$$

\section{- High order norm estimates.}

Applying the operator $\Lambda^{s}$ to equation (4.4), then one has

$$
i \Lambda^{s} E_{t}^{\epsilon}+\Lambda^{s} \mathcal{L} \nabla\left(\nabla \cdot E^{\epsilon}\right)-\alpha \Lambda^{s} \mathcal{L} \nabla \times\left(\nabla \times E^{\epsilon}\right)-\Lambda^{s} \mathcal{L}\left(n^{\epsilon} E^{\epsilon}\right)+i \Lambda^{s} \mathcal{L}\left(E^{\epsilon} \times B^{\epsilon}\right)=0 .
$$

Taking inner product of this equation with $-\Lambda^{s} \nabla\left(\nabla \cdot E^{\epsilon}\right)+\alpha \Lambda^{s} \nabla \times\left(\nabla \times E^{\epsilon}\right)$, and using the properties for $\mathcal{L}$ given by (4.5), one can obtain

$$
\begin{aligned}
\frac{d}{d t}( & \left.\left\|\Lambda^{s}\left(\nabla \cdot E^{\epsilon}\right)\right\|_{L^{2}}^{2}+\alpha\left\|\Lambda^{s}\left(\nabla \times E^{\epsilon}\right)\right\|_{L^{2}}^{2}\right) \\
= & 2 \operatorname{Im} \int_{\mathbb{R}^{d}} \mathcal{L} \Lambda^{s}\left(n^{\epsilon} E^{\epsilon}\right) \cdot\left(-\Lambda^{s} \nabla\left(\nabla \cdot \overline{E^{\epsilon}}\right)+\alpha \Lambda^{s} \nabla \times\left(\nabla \times \overline{E^{\epsilon}}\right)\right) d x \\
& +2 \operatorname{Im} i \int_{\mathbb{R}^{d}} \mathcal{L} \Lambda^{s}\left(E^{\epsilon} \times B^{\epsilon}\right) \cdot\left(\Lambda^{s} \nabla\left(\nabla \cdot \overline{E^{\epsilon}}\right)-\alpha \Lambda^{s} \nabla \times\left(\nabla \times \overline{E^{\epsilon}}\right)\right) d x \\
= & : I_{1}+I_{2} .
\end{aligned}
$$

We multiply equation (3.1b) by $\Lambda^{2 s-2} n_{t}^{\epsilon}$ and get

$$
\begin{aligned}
\frac{1}{2} \frac{d}{d t} & \left(\left\|\Lambda^{s-1} n_{t}^{\epsilon}\right\|_{L^{2}}^{2}+\left\|\Lambda^{s} n^{\epsilon}\right\|_{L^{2}}^{2}+2 \int_{\mathbb{R}^{d}} \Lambda^{s}\left|E^{\epsilon}\right|^{2} \Lambda^{s} n^{\epsilon} d x\right) \\
= & \int_{\mathbb{R}^{d}} \Lambda^{s} n^{\epsilon} \Lambda^{s}\left|E^{\epsilon}\right|_{t}^{2} d x \\
= & 2 \operatorname{Im} \int_{\mathbb{R}^{d}} \Lambda^{s} n^{\epsilon} \Lambda^{s}\left[\left(-\mathcal{L} \nabla\left(\nabla \cdot E^{\epsilon}\right)+\alpha \mathcal{L} \nabla \times\left(\nabla \times E^{\epsilon}\right)\right) \cdot \overline{E^{\epsilon}}\right] d x \\
& +2 \operatorname{Im} \int_{\mathbb{R}^{d}} \Lambda^{s} n^{\epsilon} \Lambda^{s}\left[\left(\mathcal{L}\left(n^{\epsilon} E^{\epsilon}\right)-i \mathcal{L}\left(E^{\epsilon} \times B^{\epsilon}\right)\right) \cdot \overline{E^{\epsilon}}\right] d x \\
= & : I_{3}+I_{4} .
\end{aligned}
$$


It is obvious that

$$
\begin{aligned}
\left|I_{4}\right| & \leq C\left\|n^{\epsilon}\right\|_{H^{s}}\left(\left\|n^{\epsilon}\right\|_{H^{s}}\left\|E^{\epsilon}\right\|_{H^{s+1}}^{2}+\left\|B^{\epsilon}\right\|_{H^{s}}\left\|E^{\epsilon}\right\|_{H^{s+1}}^{2}\right) \\
& \leq C\left(\left\|n^{\epsilon}\right\|_{H^{s}}^{2}+\left\|B^{\epsilon}\right\|_{H^{s}}^{2}+\left\|E^{\epsilon}\right\|_{H^{s+1}}^{2}\right)^{2} .
\end{aligned}
$$

Now we estimate $I_{1}+I_{3}$. Since

$$
\begin{aligned}
I_{1}+I_{3}= & 2 \operatorname{Im} \int_{\mathbb{R}^{d}} \Lambda^{s+1}\left(n^{\epsilon} E^{\epsilon}\right) \cdot\left(-\Lambda^{s-1} \mathcal{L} \nabla\left(\nabla \cdot \overline{E^{\epsilon}}\right)+\Lambda^{s-1} \alpha \mathcal{L} \nabla \times\left(\nabla \times \overline{E^{\epsilon}}\right)\right) d x \\
& +2 \operatorname{Im} \int_{\mathbb{R}^{d}} \Lambda^{s} n^{\epsilon} \Lambda^{s}\left[\left(-\mathcal{L} \nabla\left(\nabla \cdot E^{\epsilon}\right)+\alpha \mathcal{L} \nabla \times\left(\nabla \times E^{\epsilon}\right)\right) \cdot \overline{E^{\epsilon}}\right] d x,
\end{aligned}
$$

if we take $f=E^{\epsilon}, g=-\Lambda^{-2} \mathcal{L} \nabla\left(\nabla \cdot E^{\epsilon}\right)+\Lambda^{-2} \alpha \mathcal{L} \nabla \times\left(\nabla \times E^{\epsilon}\right), h=n^{\epsilon}$, then Lemma 4.1 below

$$
\begin{aligned}
I_{1}+I_{3} & =2 \operatorname{Im} \int_{\mathbb{R}^{d}} \Lambda^{s+1}(f h) \Lambda^{s+1} \bar{g} d x+2 \operatorname{Im} \int_{\mathbb{R}^{d}} \Lambda^{s}\left(\bar{f} \Lambda^{2} g\right) \Lambda^{s} h d x \\
& \leq C\|f\|_{H^{s+1}}\|g\|_{H^{s+1}}\|h\|_{H^{s}} \\
& \leq C\left\|E^{\epsilon}\right\|_{H^{s+1}}^{2}\left\|n^{\epsilon}\right\|_{H^{s}} \\
& \leq C\left(\left\|E^{\epsilon}\right\|_{H^{s+1}}^{2}+\left\|n^{\epsilon}\right\|_{H^{s}}^{2}+1\right)^{2} .
\end{aligned}
$$

Multiplying equation (3.1c) by $\Lambda^{2 s-4} B_{t}^{\epsilon}$, and using the fact

$$
\begin{aligned}
& \left(E^{\epsilon} \times \overline{E^{\epsilon}}\right)_{t}=2 i \operatorname{Im}\left(E_{t}^{\epsilon} \times \overline{E^{\epsilon}}\right)=-2 i \operatorname{Re}\left(i E_{t}^{\epsilon} \times \overline{E^{\epsilon}}\right) \\
& \quad=2 i \operatorname{Re}\left[\left(\mathcal{L} \nabla\left(\nabla \cdot E^{\epsilon}\right)-\alpha \mathcal{L} \nabla \times\left(\nabla \times E^{\epsilon}\right)-\mathcal{L}\left(n^{\epsilon} E^{\epsilon}\right)+i \mathcal{L}\left(E^{\epsilon} \times B^{\epsilon}\right)\right) \times \overline{E^{\epsilon}}\right],
\end{aligned}
$$

then we obtain

$$
\begin{aligned}
\frac{1}{2} \frac{d}{d t} & \left(\left\|\Lambda^{s-2} B_{t}^{\epsilon}\right\|_{L^{2}}^{2}+\left\|\Lambda^{s} B^{\epsilon}\right\|_{L^{2}}^{2}+\left\|\Lambda^{s-1} B^{\epsilon}\right\|_{L^{2}}^{2}+2 i \int_{\mathbb{R}^{d}} \Lambda^{s}\left(E^{\epsilon} \times \overline{E^{\epsilon}}\right) \Lambda^{s} B^{\epsilon} d x\right) \\
= & i \int_{\mathbb{R}^{d}} \Lambda^{s} B^{\epsilon} \Lambda^{s}\left(E^{\epsilon} \times \overline{E^{\epsilon}}\right){ }_{t} d x \\
= & -2 \operatorname{Re} \int_{\mathbb{R}^{d}} \Lambda^{s} B^{\epsilon} \Lambda^{s}\left[\left(\mathcal{L} \nabla\left(\nabla \cdot E^{\epsilon}\right)-\alpha \mathcal{L} \nabla \times\left(\nabla \times E^{\epsilon}\right)\right) \times \overline{E^{\epsilon}}\right] d x \\
& -2 \operatorname{Re} \int_{\mathbb{R}^{d}} \Lambda^{s} B^{\epsilon} \Lambda^{s}\left[\left(-\mathcal{L}\left(n^{\epsilon} E^{\epsilon}\right)+i \mathcal{L}\left(E^{\epsilon} \times B^{\epsilon}\right)\right) \times \overline{E^{\epsilon}}\right] d x \\
= & : I_{5}+I_{6} .
\end{aligned}
$$

Again the term $I_{6}$ can be estimated easily

$$
\begin{aligned}
\left|I_{6}\right| & \leq C\left\|B^{\epsilon}\right\|_{H^{s}}\left(\left\|n^{\epsilon}\right\|_{H^{s}}\left\|E^{\epsilon}\right\|_{H^{s+1}}^{2}+\left\|B^{\epsilon}\right\|_{H^{s}}\left\|E^{\epsilon}\right\|_{H^{s+1}}^{2}\right) \\
& \leq C\left(\left\|n^{\epsilon}\right\|_{H^{s}}^{2}+\left\|B^{\epsilon}\right\|_{H^{s}}^{2}+\left\|E^{\epsilon}\right\|_{H^{s+1}}^{2}\right)^{2} .
\end{aligned}
$$

We need to estimate $I_{2}+I_{5}$. Rewrite $I_{2}+I_{5}$ in the form

$$
\begin{aligned}
I_{2}+I_{5}= & -2 \operatorname{Re} \int_{\mathbb{R}^{d}} \Lambda^{s+1}\left(E^{\epsilon} \times B^{\epsilon}\right) \cdot\left(\Lambda^{s-1} \mathcal{L} \nabla\left(\nabla \cdot \overline{E^{\epsilon}}\right)-\Lambda^{s-1} \alpha \mathcal{L} \nabla \times\left(\nabla \times \overline{E^{\epsilon}}\right)\right) d x \\
& +2 \operatorname{Re} \int_{\mathbb{R}^{d}} \Lambda^{s} B^{\epsilon} \Lambda^{s}\left[\overline{E^{\epsilon}} \times\left(\mathcal{L} \nabla\left(\nabla \cdot E^{\epsilon}\right)-\alpha \mathcal{L} \nabla \times\left(\nabla \times E^{\epsilon}\right)\right)\right] d x,
\end{aligned}
$$


if we again take $f=E^{\epsilon}, g=\Lambda^{-2} \mathcal{L} \nabla\left(\nabla \cdot E^{\epsilon}\right)-\Lambda^{-2} \alpha \mathcal{L} \nabla \times\left(\nabla \times E^{\epsilon}\right), \tilde{h}=B^{\epsilon}$, then by Lemma 4.1 below, we have

$$
\begin{aligned}
I_{2}+I_{5} & =-2 \operatorname{Re} \int_{\mathbb{R}^{d}} \Lambda^{s+1}(f \times \tilde{h}) \Lambda^{s+1} \bar{g} d x+2 \operatorname{Re} \int_{\mathbb{R}^{d}} \Lambda^{s}\left(\bar{f} \times \Lambda^{2} g\right) \Lambda^{s} \tilde{h} d x \\
& \leq C\|f\|_{H^{s+1}}\|g\|_{H^{s+1}}\|\tilde{h}\|_{H^{s}} \\
& \leq C\left\|E^{\epsilon}\right\|_{H^{s+1}}^{2}\left\|B^{\epsilon}\right\|_{H^{s}} \\
& \leq C\left(\left\|E^{\epsilon}\right\|_{H^{s+1}}^{2}+\left\|B^{\epsilon}\right\|_{H^{s}}^{2}+1\right)^{2} .
\end{aligned}
$$

Combining (4.12)-(4.15) and (4.17)-(4.19) together, we arrive at

$$
\begin{aligned}
\left\|\Lambda^{s}\left(\nabla \cdot E^{\epsilon}\right)\right\|_{L^{2}}^{2}+\alpha\left\|\Lambda^{s}\left(\nabla \times E^{\epsilon}\right)\right\|_{L^{2}}^{2}+\left\|\Lambda^{s-1} n_{t}^{\epsilon}\right\|_{L^{2}}^{2}+\left\|\Lambda^{s} n^{\epsilon}\right\|_{L^{2}}^{2} & +\left\|\Lambda^{s-2} B_{t}^{\epsilon}\right\|_{L^{2}}^{2}+\left\|\Lambda^{s} B^{\epsilon}\right\|_{L^{2}}^{2}+\left\|\Lambda^{s-1} B^{\epsilon}\right\|_{L^{2}}^{2} \\
\leq & C+C \int_{0}^{t}\left(\left\|n^{\epsilon}\right\|_{H^{s}}^{2}+\left\|B^{\epsilon}\right\|_{H^{s}}^{2}+\left\|E^{\epsilon}\right\|_{H^{s+1}}^{2}\right)^{2} d \tau \\
& +\left.2\left|\int_{\mathbb{R}^{d}} \Lambda^{s}\right| E^{\epsilon}\right|^{2} \Lambda^{s} n^{\epsilon} d x|+2| \int_{\mathbb{R}^{d}} \Lambda^{s}\left(E^{\epsilon} \times \overline{E^{\epsilon}}\right) \Lambda^{s} B^{\epsilon} d x \mid .
\end{aligned}
$$

Using Cauchy-Schwarz inequality, we obtain

$$
\left.\left|\int_{\mathbb{R}^{d}} \Lambda^{s}\right| E^{\epsilon}\right|^{2} \Lambda^{s} n^{\epsilon} d x\left|\leq\left\|\Lambda^{s} n^{\epsilon}\right\|_{L^{2}}\left\|\Lambda^{s}\left|E^{\epsilon}\right|^{2}\right\|_{L^{2}} \leq \frac{1}{2}\left\|\Lambda^{s} n^{\epsilon}\right\|_{L^{2}}^{2}+\frac{1}{2}\left\|\Lambda^{s}\left|E^{\epsilon}\right|^{2}\right\|_{L^{2}}^{2}\right.
$$

and

$$
\begin{aligned}
\left|\int_{\mathbb{R}^{d}} \Lambda^{s}\left(E^{\epsilon} \times \overline{E^{\epsilon}}\right) \Lambda^{s} B^{\epsilon} d x\right| & \leq\left\|\Lambda^{s} B^{\epsilon}\right\|_{L^{2}}\left\|\Lambda^{s}\left(E^{\epsilon} \times \overline{E^{\epsilon}}\right)\right\|_{L^{2}} \\
& \leq \frac{1}{2}\left\|\Lambda^{s} B^{\epsilon}\right\|_{L^{2}}^{2}+\frac{1}{2}\left\|\Lambda^{s}\left(E^{\epsilon} \times \overline{E^{\epsilon}}\right)\right\|_{L^{2}}^{2} .
\end{aligned}
$$

Then by (4.9) we have

$$
\begin{aligned}
\frac{d}{d t} \int_{\mathbb{R}^{d}}\left(\Lambda^{s}\left|E^{\epsilon}\right|^{2}\right)^{2} d x & =2 \int_{\mathbb{R}^{d}} \Lambda^{s}\left|E^{\epsilon}\right|^{2} \Lambda^{s}\left(\left|E^{\epsilon}\right|_{t}^{2}\right) d x \\
& \leq C\left(\left\|E^{\epsilon}\right\|_{H^{s+1}}^{4}+\left\|E^{\epsilon}\right\|_{H^{s+1}}^{4}\left(\left\|n^{\epsilon}\right\|_{H^{s}}+\left\|B^{\epsilon}\right\|_{H^{s}}\right)\right) \\
& \leq C\left(\left\|E^{\epsilon}\right\|_{H^{s+1}}^{2}+\left\|n^{\epsilon}\right\|_{H^{s}}^{2}+\left\|B^{\epsilon}\right\|_{H^{s}}^{2}+1\right)^{3}
\end{aligned}
$$

which gives that

$$
\left\|\Lambda^{s}\left|E^{\epsilon}\right|^{2}\right\|_{L^{2}}^{2} \leq C+C \int_{0}^{t}\left(\left\|E^{\epsilon}\right\|_{H^{s+1}}^{2}+\left\|n^{\epsilon}\right\|_{H^{s}}^{2}+\left\|B^{\epsilon}\right\|_{H^{s}}^{2}+1\right)^{3} d \tau .
$$

Using (4.16) and the same argument, we can also get

$$
\left\|\Lambda^{s}\left(E^{\epsilon} \times \overline{E^{\epsilon}}\right)\right\|_{L^{2}}^{2} \leq C+C \int_{0}^{t}\left(\left\|E^{\epsilon}\right\|_{H^{s+1}}^{2}+\left\|n^{\epsilon}\right\|_{H^{s}}^{2}+\left\|B^{\epsilon}\right\|_{H^{s}}^{2}+1\right)^{3} d \tau .
$$


Inserting (4.21)-(4.24) into (4.20), hence we have

$$
\text { LHS of (4.20) } \leq C+C \int_{0}^{t}\left(\left\|E^{\epsilon}\right\|_{H^{s+1}}^{2}+\left\|n^{\epsilon}\right\|_{H^{s}}^{2}+\left\|B^{\epsilon}\right\|_{H^{s}}^{2}+1\right)^{3} d \tau \text {. }
$$

\section{- Conclusions.}

It concludes from the low order estimate (4.11) and the high order estimate (4.25) that

$$
\begin{gathered}
\left\|E^{\epsilon}\right\|_{H^{s+1}}^{2}+\left\|n^{\epsilon}\right\|_{H^{s}}^{2}+\left\|B^{\epsilon}\right\|_{H^{s} \cap \dot{H}^{-1}}^{2}+\left\|n_{t}^{\epsilon}\right\|_{H^{s-1} \cap \dot{H}^{-1}}^{2}+\left\|B_{t}^{\epsilon}\right\|_{H^{s-2} \cap \dot{H}^{-2}}^{2} \\
\leq C+C \int_{0}^{t}\left(\left\|E^{\epsilon}\right\|_{H^{s+1}}^{2}+\left\|n^{\epsilon}\right\|_{H^{s}}^{2}+\left\|B^{\epsilon}\right\|_{H^{s}}^{2}+1\right)^{3} d \tau,
\end{gathered}
$$

where $C$ depends on $c_{0}$, hence by Lemma 4.2 below, we know there exist $T>0$ and $C>0$ both independent of $\epsilon$ such that for all $t \in[0, T]$

$$
\left\|E^{\epsilon}\right\|_{H^{s+1}}^{2}+\left\|n^{\epsilon}\right\|_{H^{s}}^{2}+\left\|B^{\epsilon}\right\|_{H^{s} \cap \dot{H}^{-1}}^{2}+\left\|n_{t}^{\epsilon}\right\|_{H^{s-1} \cap \dot{H}^{-1}}^{2}+\left\|B_{t}^{\epsilon}\right\|_{H^{s-2} \cap \dot{H}^{-2}}^{2} \leq C,
$$

from which Proposition 4.1 follows.

Now we are going to prove the following lemma which is used in the proof of Proposition 4.1 .

Lemma 4.1. Assume that $f, g \in H^{s+1}\left(\mathbb{R}^{d}\right)$ are $\mathbb{C}^{3}$ valued functions, and $\tilde{h} \in H^{s}\left(\mathbb{R}^{d}\right)$ is a $\mathbb{R}^{3}$ valued function, and $h \in H^{s}\left(\mathbb{R}^{d}\right)$ is a real valued function, $s>\frac{d}{2}$. Then the following three estimates hold:

$$
\begin{aligned}
& \left|\operatorname{Im} \int_{\mathbb{R}^{d}} \Lambda^{s+1}(f h) \cdot \Lambda^{s+1} \bar{g} d x+\operatorname{Im} \int_{\mathbb{R}^{d}} \Lambda^{s}\left(\bar{f} \cdot \Lambda^{2} g\right) \Lambda^{s} h d x\right| \leq C\|f\|_{H^{s+1}}\|g\|_{H^{s+1}}\|h\|_{H^{s}} \\
& \left|\operatorname{Re} \int_{\mathbb{R}^{d}} \Lambda^{s+1}(f h) \cdot \Lambda^{s+1} \bar{g} d x-\operatorname{Re} \int_{\mathbb{R}^{d}} \Lambda^{s}\left(\bar{f} \cdot \Lambda^{2} g\right) \Lambda^{s} h d x\right| \leq C\|f\|_{H^{s+1}}\|g\|_{H^{s+1}}\|h\|_{H^{s}} \\
& \left|\operatorname{Re} \int_{\mathbb{R}^{d}} \Lambda^{s+1}(f \times \tilde{h}) \cdot \Lambda^{s+1} \bar{g} d x-\operatorname{Re} \int_{\mathbb{R}^{d}} \Lambda^{s}\left(\bar{f} \times \Lambda^{2} g\right) \cdot \Lambda^{s} \tilde{h} d x\right| \leq C\|f\|_{H^{s+1}}\|g\|_{H^{s+1}}\|\tilde{h}\|_{H^{s}}
\end{aligned}
$$

Proof. We first show (4.27). Denote the LHS of (4.27) by $|J|=\left|J_{1}+J_{2}\right|$. The term $J_{1}$ can be written as

$$
\begin{aligned}
J_{1}= & \operatorname{Im} \int_{\mathbb{R}^{d}}\left[\Lambda^{s+1}(f h)-f \Lambda^{s+1} h\right] \cdot \Lambda^{s+1} \bar{g} d x+\operatorname{Im} \int_{\mathbb{R}^{d}} f \Lambda^{s+1} h \cdot \Lambda^{s+1} \bar{g} d x \\
= & \operatorname{Im} \int_{\mathbb{R}^{d}}\left[\Lambda^{s+1}(f h)-f \Lambda^{s+1} h\right] \cdot \Lambda^{s+1} \bar{g} d x \\
& +\operatorname{Im} \int_{\mathbb{R}^{d}} \Lambda^{s} h\left[\Lambda\left(f \cdot \Lambda^{s+1} \bar{g}\right)-f \cdot \Lambda^{s+2} \bar{g}\right] d x+\operatorname{Im} \int_{\mathbb{R}^{d}} f \Lambda^{s} h \cdot \Lambda^{s+2} \bar{g} d x \\
= & : J_{11}+J_{12}+J_{13} .
\end{aligned}
$$


From the commutator estimate (3.8), we have

$$
\begin{aligned}
\left|J_{11}\right| & \leq C\left(\|\nabla f\|_{L^{\infty}}\|h\|_{H^{s}}+\|f\|_{H^{s+1}}\|h\|_{L^{\infty}}\right)\|g\|_{H^{s+1}} \\
& \leq C\|f\|_{H^{s+1}}\|g\|_{H^{s+1}}\|h\|_{H^{s}},
\end{aligned}
$$

since $s>\frac{d}{2}$. The term $J_{12}$ can be estimated by commutator estimate (3.8) again

$$
\left|J_{12}\right| \leq C\|h\|_{H^{s}}\left(\|\nabla f\|_{L^{\infty}}\|g\|_{H^{s+1}}+\|f\|_{H^{1, p}}\|g\|_{H^{s+1, q}}\right),
$$

where $\frac{1}{p}+\frac{1}{q}=\frac{1}{2}$. Since $s>\frac{d}{2}$, we have $H^{s+1} \hookrightarrow H^{1, p}$ for all $p \in[2, \infty]$, then

$$
\left|J_{12}\right| \leq C\|h\|_{H^{s}}\left(\|f\|_{H^{s+1}}\|g\|_{H^{s+1}}+\|f\|_{H^{s+1}}\|g\|_{H^{s+1, q}}\right),
$$

letting $q \rightarrow 2^{+}$in the above inequality, then we get

$$
\left|J_{12}\right| \leq C\|f\|_{H^{s+1}}\|g\|_{H^{s+1}}\|h\|_{H^{s}} .
$$

Now we estimate $J_{2}$. It is obvious that

$$
\begin{aligned}
J_{2} & =\operatorname{Im} \int_{\mathbb{R}^{d}}\left[\Lambda^{s}\left(\bar{f} \cdot \Lambda^{2} g\right)-\bar{f} \cdot \Lambda^{s+2} g\right] \Lambda^{s} h d x+\operatorname{Im} \int_{\mathbb{R}^{d}} \bar{f} \cdot \Lambda^{s+2} g \Lambda^{s} h d x \\
& =: J_{21}+J_{22} .
\end{aligned}
$$

Using commutator estimate (3.8), we obtain

$$
\left|J_{21}\right| \leq C\left(\|\nabla f\|_{L^{\infty}}\|g\|_{H^{s+1}}+\|f\|_{H^{s, p}}\|g\|_{H^{2, q}}\right)\|h\|_{H^{s}},
$$

where we select $p, q$ satisfying

$$
\frac{1}{p}+\frac{1}{q}=\frac{1}{2}, \frac{d}{2} \leq 1+\frac{d}{p}, 1+\frac{d}{2}-\frac{d}{q} \leq s,
$$

hence we have $H^{s+1} \hookrightarrow H^{s, p}$ and $H^{s+1} \hookrightarrow H^{2, q}$, and we therefore get

$$
\left|J_{21}\right| \leq C\|f\|_{H^{s+1}}\|g\|_{H^{s+1}}\|h\|_{H^{s}} .
$$

Since $J_{13}+J_{22}=0$, now (4.27) follows from (4.30)-(4.34). If we replace $f$ by $i \cdot f$, then (4.28) is a direct consequence of (4.27). Moreover, if we expand the term $\Lambda^{s+1}(f \times$ $\tilde{h}) \cdot \Lambda^{s+1} \bar{g}$ and $\Lambda^{s}\left(\bar{f} \times \Lambda^{2} g\right) \cdot \Lambda^{s} \tilde{h}$ by the definition of dot product and cross product, then one can easily see that (4.29) reduces to (4.28). We thus finish the proof of Lemma 4.1 .

Now we end this section with the following elementary lemma.

Lemma 4.2. Let $u(t)$ be a continuous and nonnegative function defined on $\mathbb{R}^{+}$, and suppose $u$ obeys the integral inequality

$$
u(t) \leq A_{1}+A_{2} \int_{0}^{t} u^{\kappa}(\tau) d \tau, \kappa>1
$$


for all $t \geq 0$, where $A_{1}, A_{2}>0$. Moreover, let

$$
v(t)=\frac{A_{1}}{\left(1-(\kappa-1) A_{1}^{\kappa-1} A_{2} t\right)^{1 /(\kappa-1)}},
$$

and

$$
T^{*}=\frac{1}{(\kappa-1) A_{1}^{\kappa-1} A_{2}} .
$$

Then

$$
u(t) \leq v(t), \forall t \in\left[0, T^{*}\right) .
$$

Proof. It is obviously that $v$ satisfies

$$
v(t)=A_{1}+A_{2} \int_{0}^{t} v^{\kappa}(\tau) d \tau
$$

hence this equality and (4.35) yield

$$
u(t)-v(t) \leq A_{2} \int_{0}^{t} \kappa(\theta u(\tau)+(1-\theta) v(\tau))^{\kappa-1}(u(\tau)-v(\tau)) d \tau
$$

which implies that

$$
w(t) \leq A_{2} \int_{0}^{t} \kappa(\theta u(\tau)+(1-\theta) v(\tau))^{\kappa-1} w(\tau) d \tau,
$$

where $w(t):=(u(t)-v(t))_{+}=\max \{u(t)-v(t), 0\}$. Fix any $T<T^{*}$, since both $u$ and $v$ are nonnegative and continuous, we have $0 \leq u(t), v(t) \leq K$ for all $t \in[0, T]$. Thus we obtain

$$
w(t) \leq \kappa A_{2} K^{\kappa-1} \int_{0}^{t} w(\tau) d \tau,
$$

by Gronwall's inequality, then we have $w(t)=0$ for all $t \in[0, T]$. Let $T \rightarrow T^{*}$, Lemma 4.2 thus follows.

\section{Strong convergence of the approximate solutions}

Under the prior estimates given in Proposition 4.1, we now show that the solutions $\left(E^{\epsilon}, n^{\epsilon}, B^{\epsilon}\right)$ to the regularized system (3.1a)-(3.1d) form a Cauchy sequence in the low order norm $C\left([0, T) ; H^{1} \oplus L^{2} \oplus\left(L^{2} \cap \dot{H}^{-1}\right)\right)$. Namely, we are going to prove the following lemma.

Lemma 5.1. With the same assumptions as Proposition 4.1 , then the family $\left(E^{\epsilon}, n^{\epsilon}, B^{\epsilon}\right)$ forms a Cauchy sequence in $C\left([0, T] ; H^{1} \oplus L^{2} \oplus\left(L^{2} \cap H^{-1}\right)\right)$, a.e. there holds

$$
\sup _{t \in[0, T]}\left(\left\|E^{\epsilon}-E^{\epsilon^{\prime}}\right\|_{H^{1}}+\left\|n^{\epsilon}-n^{\epsilon^{\prime}}\right\|_{L^{2}}+\left\|B^{\epsilon}-B^{\epsilon^{\prime}}\right\|_{L^{2} \cap \dot{H}^{-1}}\right) \rightarrow 0, \epsilon, \epsilon^{\prime} \rightarrow 0 .
$$

Moreover, there holds

$$
\sup _{t \in[0, T]}\left(\left\|E_{t}^{\epsilon}-E_{t}^{\epsilon^{\prime}}\right\|_{H^{-1}}+\left\|n_{t}^{\epsilon}-n_{t}^{\epsilon^{\prime}}\right\|_{\dot{H}^{-1}}+\left\|B_{t}^{\epsilon}-B_{t}^{\epsilon^{\prime}}\right\|_{\dot{H}^{-2}}\right) \rightarrow 0, \epsilon, \epsilon^{\prime} \rightarrow 0 .
$$


Proof. For brevity, we set $E=E^{\epsilon}-E^{\epsilon^{\prime}}, n=n^{\epsilon}-n^{\epsilon^{\prime}}, B=B^{\epsilon}-B^{\epsilon^{\prime}}$. Since $\left(E^{\epsilon}, n^{\epsilon}, B^{\epsilon}\right)$ and $\left(E^{\epsilon^{\prime}}, n^{\epsilon^{\prime}}, B^{\epsilon^{\prime}}\right)$ both satisfy the regularized system (3.1a)-(3.1c), then $(E, n, B)$ satisfies the equation

$$
\begin{aligned}
& i E_{t}+\mathcal{L} \nabla(\nabla \cdot E)-\alpha \mathcal{L} \nabla \times(\nabla \times E)-\mathcal{L}\left(n E^{\epsilon}+n^{\epsilon^{\prime}} E\right)+i \mathcal{L}\left(E \times B^{\epsilon}+E^{\epsilon^{\prime}} \times B\right)=0 \\
& n_{t t}-\Delta n=\Delta\left(\left|E^{\epsilon}\right|^{2}-\left|E^{\epsilon^{\prime}}\right|^{2}\right) \\
& B_{t t}+\Delta^{2} B-\Delta B=-i \Delta^{2}\left(E^{\epsilon} \times \overline{E^{\epsilon}}-E^{\epsilon^{\prime}} \times \overline{E^{\epsilon^{\prime}}}\right)
\end{aligned}
$$

with initial data

$$
\begin{aligned}
& E(0)=E_{0}^{\epsilon}-E_{0}^{\epsilon^{\prime}}, n(0)=n_{0}^{\epsilon}-n_{0}^{\epsilon^{\prime}}, n_{t}(0)=n_{1}^{\epsilon}-n_{1}^{\epsilon^{\prime}}, \\
& B(0)=B_{0}^{\epsilon}-B_{0}^{\epsilon^{\prime}}, B_{t}(0)=B_{1}^{\epsilon}-B_{1}^{\epsilon^{\prime}} .
\end{aligned}
$$

From equation (5.3a), one can obtain

$$
\begin{aligned}
\frac{d}{d t}\|E\|_{L^{2}}^{2} & =2 \operatorname{Im}\left(\mathcal{L}\left(n E^{\epsilon}+n^{\epsilon^{\prime}} E\right)-i \mathcal{L}\left(E \times B^{\epsilon}+E^{\epsilon^{\prime}} \times B\right), E\right) \\
& \leq C\left(\|n\|_{L^{2}}\|E\|_{L^{2}}+\|E\|_{L^{2}}^{2}+\|E\|_{L^{2}}\|B\|_{L^{2}}\right) \\
& \leq C\left(\|n\|_{L^{2}}^{2}+\|E\|_{L^{2}}^{2}+\|B\|_{L^{2}}^{2}\right)
\end{aligned}
$$

where we have used the prior estimate (4.2) in the first inequality above.

If we multiply equation (5.3a) by $-\nabla(\nabla \cdot \bar{E})+\alpha \nabla \times(\nabla \times \bar{E})$, and integrate the imaginary part of the result, then we have

$$
\begin{aligned}
\frac{d}{d t}\left(\|\nabla \cdot E\|_{L^{2}}^{2}+\alpha\|\nabla \times E\|_{L^{2}}^{2}\right) \\
=2 \operatorname{Im} \int_{\mathbb{R}^{d}} \mathcal{L}\left(n E^{\epsilon}\right) \cdot(-\nabla(\nabla \cdot \bar{E})+\alpha \nabla \times(\nabla \times \bar{E})) d x \\
\quad+2 \operatorname{Im} \int_{\mathbb{R}^{d}} \mathcal{L}\left(n^{\epsilon^{\prime}} E\right) \cdot(-\nabla(\nabla \cdot \bar{E})+\alpha \nabla \times(\nabla \times \bar{E})) d x \\
\quad-2 \operatorname{Im} i \int_{\mathbb{R}^{d}} \mathcal{L}\left(E \times B^{\epsilon}\right) \cdot(-\nabla(\nabla \cdot \bar{E})+\alpha \nabla \times(\nabla \times \bar{E})) d x \\
\\
\quad-2 \operatorname{Im} i \int_{\mathbb{R}^{d}} \mathcal{L}\left(E^{\epsilon^{\prime}} \times B\right) \cdot(-\nabla(\nabla \cdot \bar{E})+\alpha \nabla \times(\nabla \times \bar{E})) d x \\
=: K_{1}+K_{2}+K_{3}+K_{4} .
\end{aligned}
$$

Using integrating by parts, Sobolev inequality, and the prior estimate (4.2), we can easily get

$$
\left|K_{2}\right|+\left|K_{3}\right| \leq C\|E\|_{H^{1}}^{2} .
$$

Now taking inner product to equation (5.3b) with $\Lambda^{-2} n_{t}$, then

$$
\frac{1}{2} \frac{d}{d t}\left(\left\|\Lambda^{-1} n_{t}\right\|_{L^{2}}^{2}+\|n\|_{L^{2}}^{2}+2 \int_{\mathbb{R}^{d}}\left(\left|E^{\epsilon}\right|^{2}-\left|E^{\epsilon^{\prime}}\right|^{2}\right) \cdot n d x\right)=\int_{\mathbb{R}^{d}}\left(\left|E^{\epsilon}\right|_{t}^{2}-\left|E^{\epsilon^{\prime}}\right|_{t}^{2}\right) n d x
$$


From (4.9), we compute

$$
\begin{aligned}
\left|E^{\epsilon}\right|_{t}^{2}-\left|E^{\epsilon^{\prime}}\right|_{t}^{2}= & 2 \operatorname{Im}[(-\mathcal{L} \nabla(\nabla \cdot E)+\alpha \mathcal{L} \nabla \times(\nabla \times E) \\
& \left.\left.+\mathcal{L}\left(n E^{\epsilon}+n^{\epsilon^{\prime}} E\right)-i \mathcal{L}\left(E \times B^{\epsilon}+E^{\epsilon^{\prime}} \times B\right)\right) \cdot \overline{E^{\epsilon}}\right] \\
& +2 \operatorname{Im}\left[\left(-\mathcal{L} \nabla\left(\nabla \cdot E^{\epsilon^{\prime}}\right)+\alpha \mathcal{L} \nabla \times\left(\nabla \times E^{\epsilon^{\prime}}\right)\right.\right. \\
& \left.\left.+\mathcal{L}\left(n^{\epsilon^{\prime}} E^{\epsilon^{\prime}}\right)-i \mathcal{L}\left(E^{\epsilon^{\prime}} \times B^{\epsilon^{\prime}}\right)\right) \cdot \bar{E}\right] .
\end{aligned}
$$

Plugging this equality into (5.7), and again using the prior estimate (4.2), sometimes integrating by parts, and using Sobolev inequality, then we have

$$
\begin{aligned}
\text { LHS of (5.7) } \leq & 2 \operatorname{Im} \int_{\mathbb{R}^{d}}\left[(-\mathcal{L} \nabla(\nabla \cdot E)+\alpha \mathcal{L} \nabla \times(\nabla \times E)) \cdot \overline{E^{\epsilon}}\right] n d x \\
& +C\left(\|E\|_{H^{1}}^{2}+\|n\|_{L^{2}}^{2}+\|B\|_{L^{2}}^{2}\right) \\
& =: K_{5}+C\left(\|E\|_{H^{1}}^{2}+\|n\|_{L^{2}}^{2}+\|B\|_{L^{2}}^{2}\right) .
\end{aligned}
$$

For the equation (5.3c), we multiply it by $\Lambda^{-4} B_{t}$ and obtain

$$
\begin{aligned}
& \frac{1}{2} \frac{d}{d t}\left(\left\|\Lambda^{-2} B_{t}\right\|_{L^{2}}^{2}+\|B\|_{L^{2}}^{2}+\left\|\Lambda^{-1} B\right\|_{L^{2}}^{2}\right. \\
& \left.\quad+2 i \int_{\mathbb{R}^{d}}\left(E^{\epsilon} \times \overline{E^{\epsilon}}-E^{\epsilon^{\prime}} \times \overline{E^{\epsilon^{\prime}}}\right) \cdot B d x\right)=i \int_{\mathbb{R}^{d}}\left(E^{\epsilon} \times \overline{E^{\epsilon}}-E^{\epsilon^{\prime}} \times \overline{E^{\epsilon^{\prime}}}\right)_{t} \cdot B d x .
\end{aligned}
$$

Using (4.16), we have

$$
\begin{aligned}
& \left(E^{\epsilon} \times \overline{E^{\epsilon}}-E^{\epsilon^{\prime}} \times \overline{E^{\epsilon^{\prime}}}\right)_{t}=2 i \operatorname{Re}[(\mathcal{L} \nabla(\nabla \cdot E)-\alpha \mathcal{L} \nabla \times(\nabla \times E) \\
& \left.\left.-\mathcal{L}\left(n E^{\epsilon}+n^{\epsilon^{\prime}} E\right)+i \mathcal{L}\left(E \times B^{\epsilon}+E^{\epsilon^{\prime}} \times B\right)\right) \times \overline{E^{\epsilon^{\prime}}}\right] \\
& +2 i \operatorname{Re}\left[\left(\mathcal{L} \nabla\left(\nabla \cdot E^{\epsilon}\right)-\alpha \mathcal{L} \nabla \times\left(\nabla \times E^{\epsilon}\right)\right.\right. \\
& \left.\left.-\mathcal{L}\left(n^{\epsilon} E^{\epsilon}\right)-i \mathcal{L}\left(E^{\epsilon} \times B^{\epsilon}\right)\right) \times \bar{E}\right] .
\end{aligned}
$$

Then by the same reasonings that lead to (5.8), we have

$$
\begin{aligned}
\text { LHS of (15.9) } \leq & -2 \operatorname{Re} \int_{\mathbb{R}^{d}}\left[(\mathcal{L} \nabla(\nabla \cdot E)-\alpha \mathcal{L} \nabla \times(\nabla \times E)) \times \overline{E^{\epsilon^{\prime}}}\right] \cdot B d x \\
& +C\left(\|E\|_{H^{1}}^{2}+\|n\|_{L^{2}}^{2}+\|B\|_{L^{2}}^{2}\right) \\
& =: K_{6}+C\left(\|E\|_{H^{1}}^{2}+\|n\|_{L^{2}}^{2}+\|B\|_{L^{2}}^{2}\right) .
\end{aligned}
$$

Note that $K_{1}+K_{5}=0, K_{4}+K_{6}=0$, hence inequalities (5.4)-(5.6), (5.10) and (5.12) yield

$$
\begin{aligned}
& \frac{d}{d t}\left(\|E\|_{L^{2}}^{2}+\|\nabla \cdot E\|_{L^{2}}^{2}+\alpha\|\nabla \times E\|_{L^{2}}^{2}+\frac{1}{2}\left\|\Lambda^{-1} n_{t}\right\|_{L^{2}}^{2}+\frac{1}{2}\|n\|_{L^{2}}^{2}\right. \\
& \quad+\frac{1}{2}\left\|\Lambda^{-2} B_{t}\right\|_{L^{2}}^{2}+\frac{1}{2}\|B\|_{L^{2}}^{2}+\frac{1}{2}\left\|\Lambda^{-1} B\right\|_{L^{2}}^{2} \\
& \left.\quad+\int_{\mathbb{R}^{d}}\left(\left|E^{\epsilon}\right|^{2}-\left|E^{\epsilon^{\prime}}\right|^{2}\right) \cdot n d x+i \int_{\mathbb{R}^{d}}\left(E^{\epsilon} \times \overline{E^{\epsilon}}-E^{\epsilon^{\prime}} \times \overline{E^{\epsilon^{\prime}}}\right) \cdot B d x\right) \\
& \quad \leq C\left(\|E\|_{H^{1}}^{2}+\|n\|_{L^{2}}^{2}+\|B\|_{L^{2}}^{2}\right) .
\end{aligned}
$$


By integrating this inequality, we obtain

$$
\begin{aligned}
& \|E\|_{H^{1}}^{2}+\|n\|_{L^{2}}^{2}+\|B\|_{L^{2} \cap \dot{H}^{-1}}^{2}+\left\|n_{t}\right\|_{\dot{H}^{-1}}^{2}+\left\|B_{t}\right\|_{\dot{H}^{-2}}^{2} \\
& \leq C\left(\|E(0)\|_{H^{1}}^{2}+\|n(0)\|_{L^{2}}^{2}+\|B(0)\|_{L^{2} \cap \dot{H}^{-1}}^{2}+\left\|n_{t}(0)\right\|_{\dot{H}^{-1}}^{2}+\left\|B_{t}(0)\right\|_{\dot{H}^{-2}}^{2}\right) \\
& \quad+C\left|\int_{\mathbb{R}^{d}}\left(\left|E^{\epsilon}\right|^{2}-\left|E^{\epsilon^{\prime}}\right|^{2}\right) \cdot n d x\right|+C\left|\int_{\mathbb{R}^{d}}\left(E^{\epsilon} \times \overline{E^{\epsilon}}-E^{\epsilon^{\prime}} \times \overline{E^{\prime}}\right) \cdot B d x\right| \\
& \quad+C \int_{0}^{t}\left(\|E\|_{H^{1}}^{2}+\|n\|_{L^{2}}^{2}+\|B\|_{L^{2}}^{2}\right) d \tau .
\end{aligned}
$$

Applying Cauchy-Schwarz inequality, we have

$$
\begin{aligned}
& \left|\int_{\mathbb{R}^{d}}\left(\left|E^{\epsilon}\right|^{2}-\left|E^{\epsilon^{\prime}}\right|^{2}\right) \cdot n d x\right| \leq \frac{1}{2}\|n\|_{L^{2}}^{2}+\frac{1}{2}\left\|\left|E^{\epsilon}\right|^{2}-\left|E^{\epsilon^{\prime}}\right|^{2}\right\|_{L^{2}}^{2}, \\
& \left|\int_{\mathbb{R}^{d}}\left(E^{\epsilon} \times \overline{E^{\epsilon}}-E^{\epsilon^{\prime}} \times \overline{E^{\epsilon^{\prime}}}\right) \cdot B d x\right| \leq \frac{1}{2}\|B\|_{L^{2}}^{2}+\frac{1}{2}\left\|E^{\epsilon} \times \overline{E^{\epsilon}}-E^{\epsilon^{\prime}} \times \overline{E^{\epsilon^{\prime}}}\right\|_{L^{2}}^{2} .
\end{aligned}
$$

We deduce from (5.8) that

$$
\begin{aligned}
\frac{d}{d t}\left\|\left|E^{\epsilon}\right|^{2}-\left|E^{\epsilon^{\prime}}\right|^{2}\right\|_{L^{2}}^{2} & =2 \int_{\mathbb{R}^{d}}\left(\left|E^{\epsilon}\right|^{2}-\left|E^{\epsilon^{\prime}}\right|^{2}\right) \cdot\left(\left|E^{\epsilon}\right|_{t}^{2}-\left|E^{\epsilon^{\prime}}\right|_{t}^{2}\right) d x \\
& \leq C\left(\|E\|_{H^{1}}^{2}+\|n\|_{L^{2}}^{2}+\|B\|_{L^{2}}^{2}\right),
\end{aligned}
$$

thus one can obtain from this inequality

$$
\left\|\left|E^{\epsilon}\right|^{2}-\left|E^{\epsilon^{\prime}}\right|^{2}\right\|_{L^{2}}^{2} \leq C\|E(0)\|_{H^{1}}^{2}+C \int_{0}^{t}\left(\|E\|_{H^{1}}^{2}+\|n\|_{L^{2}}^{2}+\|B\|_{L^{2}}^{2}\right) d \tau .
$$

A similar argument yields(we shall use (5.11) instead)

$$
\left\|E^{\epsilon} \times \overline{E^{\epsilon}}-E^{\epsilon^{\prime}} \times \overline{E^{\epsilon^{\prime}}}\right\|_{L^{2}}^{2} \leq C\|E(0)\|_{H^{1}}^{2}+C \int_{0}^{t}\left(\|E\|_{H^{1}}^{2}+\|n\|_{L^{2}}^{2}+\|B\|_{L^{2}}^{2}\right) d \tau .
$$

Putting (5.13)-(5.16) together gives

$$
\begin{aligned}
& \|E\|_{H^{1}}^{2}+\|n\|_{L^{2}}^{2}+\|B\|_{L^{2} \cap \dot{H}^{-1}}^{2}+\left\|n_{t}\right\|_{\dot{H}^{-1}}^{2}+\left\|B_{t}\right\|_{\dot{H}^{-2}}^{2} \\
& \leq C\left(\|E(0)\|_{H^{1}}^{2}+\|n(0)\|_{L^{2}}^{2}+\|B(0)\|_{L^{2} \cap \dot{H}^{-1}}^{2}+\left\|n_{t}(0)\right\|_{\dot{H}^{-1}}^{2}+\left\|B_{t}(0)\right\|_{\dot{H}^{-2}}^{2}\right) \\
& \quad+C \int_{0}^{t}\left(\|E\|_{H^{1}}^{2}+\|n\|_{L^{2}}^{2}+\|B\|_{L^{2}}^{2}\right) d \tau .
\end{aligned}
$$

Since (4.1) holds, by Gronwall's inequality, we thus deduce from (5.17) that $\left(E^{\epsilon}, n^{\epsilon}, B^{\epsilon}\right)$ is a Cauchy sequence in $C\left([0, T] ; H^{1} \oplus L^{2} \oplus\left(L^{2} \cap \dot{H}^{-1}\right)\right)$. Moreover, from (5.17) and the equation (4.4), we get (5.2). Then Lemma 5.1 follows.

Remark 5.1. From (4.2), (4.3), (5.1), (5.2) and the following interpolation in Sobolev spaces

$$
\|f\|_{\dot{H}^{s_{0}}} \leq C\|f\|_{\dot{H}^{s_{1}}}^{1-\theta}\|f\|_{\dot{H}^{s_{2}}}^{\theta}, s_{1}<s_{2}, s_{1} \leq s_{0} \leq s_{2}, \theta=\frac{s_{0}-s_{1}}{s_{2}-s_{1}}
$$


we have as $\epsilon, \epsilon^{\prime} \rightarrow 0$

$$
\begin{aligned}
& \sup _{t \in[0, T]}\left(\left\|E^{\epsilon}-E^{\epsilon^{\prime}}\right\|_{H^{\tilde{s}+1}}+\left\|n^{\epsilon}-n^{\epsilon^{\prime}}\right\|_{H^{\tilde{s}}}+\left\|B^{\epsilon}-B^{\epsilon^{\prime}}\right\|_{H^{\tilde{s}} \cap \dot{H}^{-1}}\right) \rightarrow 0, \\
& \sup _{t \in[0, T]}\left(\left\|E_{t}^{\epsilon}-E_{t}^{\epsilon^{\prime}}\right\|_{H^{\tilde{s}-1}}+\left\|n_{t}^{\epsilon}-n_{t}^{\epsilon^{\prime}}\right\|_{H^{\tilde{s}-1} \cap \dot{H}^{-1}}+\left\|B_{t}^{\epsilon}-B_{t}^{\epsilon^{\prime}}\right\|_{H^{\tilde{s}-2} \cap \dot{H}^{-2}}\right) \rightarrow 0,
\end{aligned}
$$

for all $\tilde{s}<s$.

\section{Proof of the main theorem}

Proof of Theorem 1.1. For given $E_{0} \in H^{s+1},\left(n_{0}, n_{1}\right) \in H^{s} \oplus\left(H^{s-1} \cap \dot{H}^{-1}\right),\left(B_{0}, B_{1}\right) \in$ $\left(H^{s} \cap \dot{H}^{-1}\right) \oplus\left(H^{s-2} \cap \dot{H}^{-2}\right), s>\frac{d}{2}$, we choose $\left(E_{0}^{\epsilon}, n_{0}^{\epsilon}, n_{1}^{\epsilon}, B_{0}^{\epsilon}, B_{1}^{\epsilon}\right)$ sufficiently regular such that (4.1) holds. Then by the strong convergence results (5.18), we know that there exists $(E, n, B)$ satisfying $(\epsilon \rightarrow 0)$

$$
\begin{aligned}
& \sup _{t \in[0, T]}\left(\left\|E^{\epsilon}-E\right\|_{H^{\tilde{s}+1}}+\left\|n^{\epsilon}-n\right\|_{H^{\tilde{s}}}+\left\|B^{\epsilon}-B\right\|_{H^{\tilde{s} \cap \dot{H}^{-1}}}\right) \rightarrow 0, \\
& \sup _{t \in[0, T]}\left(\left\|E_{t}^{\epsilon}-E_{t}\right\|_{H^{\tilde{s}-1}}+\left\|n_{t}^{\epsilon}-n_{t}\right\|_{H^{\tilde{s}-1} \cap \dot{H}^{-1}}+\left\|B_{t}^{\epsilon}-B_{t}\right\|_{H^{\tilde{s}-2} \cap \dot{H}^{-2}}\right) \rightarrow 0,
\end{aligned}
$$

for all $\tilde{s}<s$. Moreover, for all $\tilde{s}<s$, we have

$$
\begin{aligned}
& n^{\epsilon} E^{\epsilon} \rightarrow n E \text { in } C\left([0, T] ; H^{\tilde{s}}\right), E^{\epsilon} \times B^{\epsilon} \rightarrow E \times B, \text { in } C\left([0, T] ; H^{\tilde{s}}\right), \\
& |E|^{2} \rightarrow|E|^{2} \text { in } C\left([0, T] ; H^{\tilde{s}+1}\right), E^{\epsilon} \times \overline{E^{\epsilon}} \rightarrow E \times \bar{E} \text {, in } C\left([0, T] ; H^{\tilde{s}+1}\right) .
\end{aligned}
$$

Now letting $\epsilon \rightarrow 0$ in (3.1a)-(3.1d), and using the above strong convergence properties, we finally see that $(E, n, B)$ is a solution of the original magnetic Zakharov system (1.1)(1.2). Furthermore, by the boundedness property (4.2)-(4.3) and the strong convergence property (6.1), we have

$$
\begin{gathered}
(E, n, B) \in C\left([0, T] ; H^{s+1} \oplus H^{s} \oplus\left(H^{s} \cap \dot{H}^{-1}\right)\right) \\
\left(E_{t}, n_{t}, B_{t}\right) \in C\left([0, T] ; H^{s-1} \oplus\left(H^{s-1} \cap \dot{H}^{-1}\right) \oplus\left(H^{s-2} \cap \dot{H}^{-2}\right)\right) .
\end{gathered}
$$

From Proposition 4.1, the existence time $T$ depends on the norm of the initial data. In fact, if $T_{\max }$ is the maximal lifespan of the solution, then either $T_{\max }=\infty$ or $T_{\max }<\infty$ and

$$
\|E(t)\|_{H^{s+1}}+\|n(t)\|_{H^{s}}+\left\|n_{t}(t)\right\|_{H^{s-1} \cap \dot{H}^{-1}}+\|B(t)\|_{H^{s} \cap \dot{H}^{-1}}+\left\|B_{t}(t)\right\|_{H^{s-2} \cap \dot{H}^{-2}} \rightarrow \infty
$$

as $t \rightarrow T_{\max }$. Hence, the local existence part of Theorem 1.1 is proved.

For the uniqueness of the system (1.1)-(1.2), one can apply the same methods used in Lemma 5.1, hence, the proof of uniqueness is essentially the same as the proof of (5.1), and we omit the details. We thus finish the proof of Theorem 1.1.

Remark 6.1. Taking $\epsilon \rightarrow 0$ in (3.2) and (3.3), and using (4.2)-(4.3), (6.1), then we see that the solution of (1.1) also satisfies the conservation laws (2.1)-(2.2). Besides, the solution $(E, n, B)$ obtained in Theorem 1.1 depends continuously on the initial data in 
the following sense: There exists $T_{1}>0$ depending on $R$ such that if $E_{0}^{j} \rightarrow E_{0}$ in $H^{s+1}, n_{0}^{j} \rightarrow n_{0}$ in $H^{s}, n_{1}^{j} \rightarrow n_{1}$ in $H^{s-1} \cap \dot{H}^{-1}, B_{0}^{j} \rightarrow B_{0}$ in $H^{s} \cap \dot{H}^{-1}, B_{1}^{j} \rightarrow B_{1}$ in $H^{s-2} \cap \dot{H}^{-2}$, and if $\left(E^{j}, n^{j}, B^{j}\right)$ is the corresponding solution of (1.1) with initial data $\left(E_{0}^{j}, n_{0}^{j}, n_{1}^{j}, B_{0}^{j}, B_{1}^{j}\right)$, then $\left(E^{j}, n^{j}, B^{j}\right)$ is defined on $\left[0, T_{1}\right]$ when $j$ is large, and

$$
\begin{aligned}
& \sup _{t \in\left[0, T_{1}\right]}\left(\left\|E^{j}-E\right\|_{H^{\tilde{s}+1}}+\left\|n^{j}-n\right\|_{H^{\tilde{s}}}+\left\|B^{j}-B\right\|_{H^{\tilde{s} \cap \dot{H}^{-1}}}\right) \rightarrow 0, \\
& \sup _{t \in\left[0, T_{1}\right]}\left(\left\|E_{t}^{j}-E_{t}\right\|_{H^{\tilde{s}-1}}+\left\|n_{t}^{j}-n_{t}\right\|_{H^{\tilde{s}-1} \cap \dot{H}^{-1}}+\left\|B_{t}^{j}-B_{t}\right\|_{H^{\tilde{s}-2} \cap \dot{H}^{-2}}\right) \rightarrow 0
\end{aligned}
$$

for all $\tilde{s}<s$ as $j \rightarrow \infty$.

Note that Theorem 1.1 needs the additional assumption $n_{1}, B_{0} \in \dot{H}^{-1}, B_{1} \in \dot{H}^{-2}$. As described in Section 1, this assumption is rather strong. In fact, this condition can be removed by splitting the initial data into low frequency part and high frequency part. Denote $\varphi(\xi) \in C_{c}^{\infty}\left(\mathbb{R}^{d}\right)$ such that $0 \leq \varphi(\xi)=\varphi(|\xi|) \leq 1, \varphi \equiv 1$ if $|\xi| \leq 1$ and $\varphi \equiv 0$ if $|\xi| \geq 2$. For any given $f \in H^{r}\left(\mathbb{R}^{d}\right), r \in \mathbb{R}$, we decompose $f=f_{L}+f_{H}$, where

$$
\widehat{f_{L}}=\varphi(\xi) \hat{f}, \widehat{f_{H}}=(1-\varphi(\xi)) \hat{f} .
$$

So one can easily see that $f_{L} \in H^{k}$ for all $k \in \mathbb{R}$ and $f_{H} \in \dot{H}^{l} \cap H^{l}$ for all $l \leq r$. Furthermore, there holds

$$
\left\|f_{L}\right\|_{H^{k}} \leq \max \left\{5^{\frac{k-r}{2}},\right\}\|f\|_{H^{r}},\left\|f_{H}\right\|_{H^{l} \cap \dot{H}^{l}} \leq \max \left\{2^{-\frac{r}{2}}, 1\right\}\|f\|_{H^{r}} .
$$

In this way, we can decompose $n_{1} \in H^{s}$ as $n_{1}=n_{1 L}+n_{1 H}$ with $n_{1 L} \in H^{k}$ for all $k \in \mathbb{R}$, $n_{1 H} \in H^{l} \cap \dot{H}^{l}$ for all $l \leq s$, and in particular $n_{1 H} \in H^{s} \cap \dot{H}^{-1}$. Moreover we have

$$
\left\|n_{1 L}\right\|_{H^{k}} \leq C(k, s)\left\|n_{1}\right\|_{H^{s}}, \quad \forall k \in \mathbb{R},\left\|n_{1 H}\right\|_{H^{s} \cap \dot{H}^{-1}} \leq\left\|n_{1}\right\|_{H^{s}} .
$$

Similarly, for given $B_{0} \in H^{s}, B_{1} \in H^{s-2}$, we have $B_{0}=B_{0 L}+B_{0 H}, B_{1}=B_{1 L}+B_{1 H}$, where $B_{0 L}, B_{1 L} \in H^{k}(k \in \mathbb{R}), B_{0 H} \in H^{s_{1}} \cap \dot{H}^{s_{1}}$ for all $s_{1} \leq s, B_{1 H} \in H^{s_{2}} \cap \dot{H}^{s_{2}}$ for all $s_{2} \leq s-2$ and

$$
\begin{aligned}
& \left\|B_{0 L}\right\|_{H^{k}} \leq C(k, s)\left\|B_{0}\right\|_{H^{s}},\left\|B_{1 L}\right\|_{H^{k}} \leq C(k, s-2)\left\|B_{1}\right\|_{H^{s-2}}, \forall k \in \mathbb{R}, \\
& \left\|B_{0 H}\right\|_{H^{s} \cap \dot{H}^{-1}} \leq\left\|B_{0}\right\|_{H^{s}},\left\|B_{1 H}\right\|_{H^{s-2} \cap \dot{H}^{-2}} \leq C(s-2)\left\|B_{0}\right\|_{H^{s-2}} .
\end{aligned}
$$

Now we set

$$
\tilde{n}=n-t n_{1 L}, \tilde{B}=B-B_{0 L}-t B_{1 L},
$$

and consider the equation

$$
\left\{\begin{array}{l}
i E_{t}+\nabla(\nabla \cdot E)-\alpha \nabla \times(\nabla \times E)-\left(\tilde{n}+t n_{1 L}\right) E+i E \times\left(\tilde{B}+B_{0 L}+t B_{1 L}\right)=0 \\
\tilde{n}_{t t}-\Delta \tilde{n}=\Delta|E|^{2}+t \Delta n_{1 L} \\
\tilde{B}_{t t}+\Delta^{2} \tilde{B}-\Delta \tilde{B}=-i \Delta^{2}(E \times \bar{E})-\Delta^{2}\left(B_{0 L}+t B_{1 L}\right)+\Delta\left(B_{0 L}+t B_{1 L}\right)
\end{array}\right.
$$

with initial data

$$
E(0, x)=E_{0}, \quad\left(\tilde{n}(0, x), \tilde{n}_{t}(0, x)\right)=\left(n_{0}, n_{1 H}\right), \quad\left(\tilde{B}(0, x), \tilde{B}_{t}(0, x)\right)=\left(B_{0 H}, B_{1 H}\right) .
$$


Note that the initial data (6.6) satisfies the condition $\tilde{n}_{t}(0) \in \dot{H}^{-1}, \tilde{B}(0) \in \dot{H}^{-1}, \tilde{B}_{t}(0) \in$ $\dot{H}^{-2}$. We also remark that if $(E, n, B)$ solves (1.1)-(1.2), then $(E, \tilde{n}, \tilde{B})$ defined by (6.4) solves (6.5)-(6.6), and vice versa.

For the regular solution of equation (6.4), a similar argument as in Proposition 2.1 gives that $\|E(t)\|_{L^{2}}=\left\|E_{0}\right\|_{L^{2}}$ and

$$
\begin{aligned}
& \frac{d}{d t}\left(\|\nabla \cdot E(t)\|_{L^{2}}^{2}+\alpha\|\nabla \times E(t)\|_{L^{2}}^{2}+\frac{1}{2}\|\tilde{n}(t)\|_{L^{2}}^{2}+\frac{1}{2}\left\|\Lambda^{-1} \tilde{n}_{t}(t)\right\|_{L^{2}}^{2}+\frac{1}{2}\left\|\Lambda^{-2} \tilde{B}_{t}(t)\right\|_{L^{2}}^{2}\right. \\
& \quad+\frac{1}{2}\|\tilde{B}(t)\|_{L^{2}}^{2}+\frac{1}{2}\left\|\Lambda^{-1} \tilde{B}(t)\right\|_{L^{2}}^{2}+\int_{\mathbb{R}^{d}} \tilde{n}(t)|E(t)|^{2} d x+\int_{\mathbb{R}^{d}} t n_{1 L}|E(t)|^{2} d x \\
& \left.\quad+i \int_{\mathbb{R}^{d}}(E(t) \times \overline{E(t)}) \cdot \tilde{B}(t) d x+i \int_{\mathbb{R}^{d}}(E(t) \times \overline{E(t)}) \cdot\left(B_{0 L}+t B_{1 L}\right) d x\right) \\
& =\int_{\mathbb{R}^{d}} n_{1 L}|E(t)|^{2} d x+i \int_{\mathbb{R}^{d}} B_{1 L} \cdot(E(t) \times \overline{E(t)}) d x-t \int_{\mathbb{R}^{d}} \Lambda n_{1 L} \Lambda^{-1} \tilde{n}_{t} d x \\
& \quad-\int_{\mathbb{R}^{d}} \Lambda^{2}\left(B_{0 L}+t B_{1 L}\right) \Lambda^{-2} \tilde{B}_{t} d x-\int_{\mathbb{R}^{d}}\left(B_{0 L}+t B_{1 L}\right) \Lambda^{-2} \tilde{B}_{t} d x .
\end{aligned}
$$

Using (6.2), (6.3) and the fact $\|E\|_{L^{2}}=\left\|E_{0}\right\|_{L^{2}}$, we have

$$
\text { RHS of (6.7) } \leq C+C(1+t)\left(\left\|\Lambda^{-1} \tilde{n}_{t}\right\|_{L^{2}}^{2}+\left\|\Lambda^{-2} \tilde{B}_{t}\right\|_{L^{2}}^{2}\right)
$$

and

$$
\begin{aligned}
& \left.\left|\int_{\mathbb{R}^{d}} t n_{1 L}\right| E(t)\right|^{2} d x \mid \leq t\left\|n_{1 L}\right\|_{L^{\infty}}\left\|E_{0}\right\|_{L^{2}}^{2} \leq C t, \\
& \left|i \int_{\mathbb{R}^{d}}(E(t) \times \overline{E(t)}) \cdot\left(B_{0 L}+t B_{1 L}\right) d x\right| \leq C(1+t) .
\end{aligned}
$$

Integrating (6.7), and applying the same method given in Lemma 2.2 and Gronwall's inequality, we can bound the quantity

$$
\|E\|_{H^{1}}^{2}+\|\tilde{n}\|_{L^{2}}^{2}+\left\|\tilde{n}_{t}\right\|_{\dot{H}^{-1}}^{2}+\|\tilde{B}\|_{L^{2} \cap \dot{H}^{-1}}^{2}+\left\|\tilde{B}_{t}\right\|_{\dot{H}^{-2}}^{2}
$$

by the norm of initial data (6.6). If we return to our original system, then we can obtain the following result.

Lemma 6.1. Assume $(E, n, B)$ is a sufficiently regular solution to the magnetic Zakharov system (1.1)-(1.2), and let $2\left\|E_{0}\right\|_{L^{2}}^{2}<\|Q\|_{L^{2}}^{2}$ in the case $d=2$, where $Q=Q(x)$ is the ground state solution of

$$
\Delta Q-Q+Q^{3}=0, x \in \mathbb{R}^{2},
$$

and $\left\|E_{0}\right\|_{H^{1}}$ small when $d=3$. Then

$$
\|E\|_{H^{1}}^{2}+\|n\|_{L^{2}}^{2}+\left\|n_{t}\right\|_{H^{-1}}^{2}+\|B\|_{L^{2}}^{2}+\left\|B_{t}\right\|_{H^{-2}}^{2} \leq C,
$$

here the constant $C$ depends on $t,\left\|E_{0}\right\|_{H^{1}},\left\|n_{0}\right\|_{L^{2}},\left\|n_{1}\right\|_{H^{-1}},\left\|B_{0}\right\|_{L^{2}},\left\|B_{1}\right\|_{H^{-2}}$.

Again Lemma 6.1 implies the existence of weak solution for the magnetic system. 
Theorem 6.2. If $E_{0} \in H^{1},\left(n_{0}, n_{1}\right) \in L^{2} \oplus H^{-1},\left(B_{0}, B_{1}\right) \in L^{2} \oplus H^{-2}$, and the initial data satisfying $2\left\|E_{0}\right\|_{L^{2}}^{2}<\|Q\|_{L^{2}}^{2}$ in the case $d=2$ and $\left\|E_{0}\right\|_{H^{1}}$ small in $d=3$, then there exists a weak solution $(E, n, B)$ for the system (1.1) in the sense of distributions such that

$$
E \in L_{l o c}^{\infty}\left(\mathbb{R}^{+} ; H^{1}\right),\left(n, n_{t}\right) \in L_{l o c}^{\infty}\left(\mathbb{R}^{+} ; L^{2} \oplus H^{-1}\right),\left(B, B_{t}\right) \in L_{l o c}^{\infty}\left(\mathbb{R}^{+} ; L^{2} \oplus H^{-2}\right) .
$$

Due to (6.2) and (6.3), we see that the low frequency part of $n_{1}, B_{0}$ and $B_{1}$ appearing in the equation (6.5) can be well controlled. Therefore, one can follow the same procedure as in Section 3-Section 5 and then get the existence and uniqueness of solution for the equation (6.5) with initial data (6.6), which in turn leads to Theorem 1.2. Since this process is much the same as the proof of Theorem 1.1, the details are omitted. Hence, in this way, Theorem 1.2 is proved.

\section{References}

[1] Added, H., Added, S. (1984). Existence globle de solutions fortes pour les équations de la turbulence de Langmuir en dimension 2. C. R. Acad. Sci. Paris 299: 551-554.

[2] Added, H., Added, S. (1988). Equations of Langmuir turbulence and nonlinear Schrödinger equation: smoothness and approximation. J. Funct. Anal. 79 (1): 183210.

[3] Bourgain, J., Colliander, J. (1996). On wellposedness of the Zakharov system. Internat. Math. Res. Notices 11: 515-546.

[4] Bejenaru, I., Herr, S., Holmer, J., Tataru, D. (2009). On the 2d Zakharov system with $L^{2}$ Schrödinger data. Nonlinearity 22: 1063-1089.

[5] Colliander, J., Holmer, J., Tzirakis, N. (2008). Low regularity global well-posedness for the Zakharov and Klein-Gordon-Schrödinger systems. Trans. of the AMS 360 (9): 4619-4638.

[6] Coifman, R., Meyer, Y. (1986). Nonlinear harmonic analysis operator theory and P.D.E., in "Beijing Lectures in Harmonic Analysis". Princeton University Press.

[7] Glangetas, L., Merle, F. (1994). Concentration properties of blow-up solutions and instability results for Zakharov equation in dimension two. Part II. Commun. Math. Phys. 160: 349-389.

[8] Guo, B., Shen, l. (1982). The existence and uniqueness of the classical solution on the periodic initial value problem for Zakharov equation. (in Chinese) Acta Mathematicae Applicatae Sinica 5 (3): 310-324.

[9] Ginibre, J., Tsutsumi, Y., Velo, G. (1997). On the Cauchy problem for the Zakharov system. J. Funct. Anal. 151 (2): 384-436. 
[10] He, X. (1983). The pondermotive force and magnetic field generation effects resulting from the non-linear interaction between plasma-wave and particles(in Chinese). Acta Physica Sinica 32 (3): 325-337.

[11] Holmer, J. (2007). Local ill-posedness of the 1D Zakharov system. Electron. J. Differential Equations 24: 22 pp.

[12] Kato, T. (1990). Liapunov functions and monotonicity in the Euler and NavierStokes equations. Lecture Notes in Mathematics 1450, Berlin: Springer-Verlag.

[13] Kono, M., Skoric, M. M., Haar, D. Ter (1981). Spontaneous excitation of magnetic fields and collapse dynamics in a Langmuir plasma. J. Plasma Phys. 26: 123-146.

[14] Laurey, C. (1995). The Cauchy problem for a generalized Zakharov system. Differential Integral Equations 8 (1): 105-130.

[15] Ozawa, T., Tsutsumi, Y. (1992). Existence and smooth effect of solutions for the Zakharov equations. Pub. RIMS. Kyoto Univ. 28: 329-361.

[16] Pecher, H. (2005). Global solutions with infinite energy for the one-dimensional Zakharov system. Electron. J. Differential Equations 41: 18 pp.

[17] Sulem, C., Sulem, P. L. (1979). Quelques résulatats de régularité pour les équation de la turbulence de Langmuir. C. R. Acad. Sci. Paris 289: 173-176.

[18] Schochet, S. H., Weinstein, M. I. (1986). The nonlinear Schrödinger limit of the Zakharov equations governing Langmuir turbulence. Commun. Math. Phys. 106: 569-580.

[19] Weinstein, M. I. (1983). Nonlinear Schrödinger equations and sharp interpolation estimates. Commun. Math. Phys. 87: 567-576.

[20] Zakharov, V. E. (1972). Collapse of Langmuir waves. Sov. Phys. JETP 35: 908-914. 